\title{
Evolution of flare ribbons, electric currents, and quasi-separatrix layers during an X-class flare ${ }^{\star}$
}

\author{
M. Janvier ${ }^{1,2}$, A. Savcheva ${ }^{3}$, E. Pariat ${ }^{4}$, S. Tassev ${ }^{3}$, S. Millholland ${ }^{5}$, V. Bommier ${ }^{4}$, P. McCauley ${ }^{3,6}$, \\ S. McKillop ${ }^{3}$, and F. Dougan ${ }^{2}$
}

\author{
${ }^{1}$ Institut d'Astrophysique Spatiale, CNRS, Univ. Paris-Sud, Université Paris-Saclay, Bât. 121, 91405 Orsay Cedex, France \\ e-mail: miho.janvier@ias.u-psud.fr \\ 2 University of Dundee, School of Science and Engineering, DD1 4HN, Dundee, UK \\ 3 Harvard-Smithsonian Astrophysical Observatory, 6a Garden Street, Cambridge, MA 02143, USA \\ ${ }^{4}$ LESIA, Observatoire de Paris, PSL Research University, CNRS, Sorbonne Universités, UPMC Univ. Paris 06, Univ. Paris Diderot, \\ Sorbonne Paris Cité, 5 place Jules Janssen, 92195 Meudon, France \\ 5 University of California, Santa Cruz, 1156 High Street, Santa Cruz, CA 95064, USA \\ ${ }^{6}$ University of Sydney, New South Wales 2006, Australia
}

Received 29 February 2016/ Accepted 21 April 2016

\begin{abstract}
Context. The standard model for eruptive flares has been extended to three dimensions (3D) in the past few years. This model predicts typical $J$-shaped photospheric footprints of the coronal current layer, forming at similar locations as the quasi-separatrix layers (QSLs). Such a morphology is also found for flare ribbons observed in the extreme ultraviolet (EUV) band, and in nonlinear force-free field (NLFFF) magnetic field extrapolations and models.

Aims. We study the evolution of the photospheric traces of the current density and flare ribbons, both obtained with the Solar Dynamics Observatory instruments. We aim to compare their morphology and their time evolution, before and during the flare, with the topological features found in a NLFFF model.

Methods. We investigated the photospheric current evolution during the 06 September 2011 X-class flare (SOL2011-09-06T22:20) occurring in NOAA AR 11283 from observational data of the magnetic field obtained with the Helioseismic and Magnetic Imager aboard the Solar Dynamics Observatory. We compared this evolution with that of the flare ribbons observed in the EUV filters of the Atmospheric Imager Assembly. We also compared the observed electric current density and the flare ribbon morphology with that of the QSLs computed from the flux rope insertion method-NLFFF model.

Results. The NLFFF model shows the presence of a fan-spine configuration of overlying field lines, due to the presence of a parasitic polarity, embedding an elongated flux rope that appears in the observations as two parts of a filament. The QSL signatures of the fan configuration appear as a circular flare ribbon that encircles the $J$-shaped ribbons related to the filament ejection. The QSLs, evolved via a magnetofrictional method, also show similar morphology and evolution as both the current ribbons and the EUV flare ribbons obtained several times during the flare.

Conclusions. For the first time, we propose a combined analysis of the photospheric traces of an eruptive flare, in a complex topology, with direct measurements of electric currents and QSLs from observational data and a magnetic field model. The results, obtained by two different and independent approaches 1) confirm previous results of current increase during the impulsive phase of the flare and 2) show how NLFFF models can capture the essential physical signatures of flares even in a complex magnetic field topology.
\end{abstract}

Key words. Sun: flares - Sun: magnetic fields - magnetic reconnection - magnetohydrodynamics (MHD)

\section{Introduction}

Solar flares are energetic events taking place in the atmosphere of our star that are characterized by a rapid increase in light emission in a wide range of the electromagnetic spectrum (Fletcher et al. 2011). In particular, eruptive flares are associated with a sudden release of solar plasma and magnetic field under the form of coronal mass ejections (CMEs), which can impact planetary environments (see Gosling et al. 1991; Gonzalez et al. 1999; Prangé et al. 2004). As such, understanding the mechanisms behind solar flares is of primary importance to better predict the influence of the Sun on its surroundings.

The underlying mechanism of solar flares is believed to be the reconnection of magnetic field lines, which is a process

\footnotetext{
* A movie associated to Fig. 1 is available in electronic form at http://www . aanda.org
}

that can accelerate particles to high energy and convert magnetic energy into kinetic energy and heat. The description of this phenomenon has been refined over the years to incorporate many of the observed flare features (see, e.g., the reviews of Priest \& Forbes 2002; Fletcher et al. 2011 and references therein).

One of these flare features in particular is the formation of two elongated, ribbon-like structures appearing during eruptive flares (see Chen 2011, and references therein). These structures emit in the extreme ultraviolet (EUV) and up to visible wavelengths and are sometimes associated with hard X-ray (HXR) sources (Emslie et al. 2003). It has been proposed that flare ribbons are formed by the collisions between high energetic particles launched from the coronal reconnection site and the chromospheric plasma, as these are locations of intense energy deposition (Hudson et al. 2006). Flare ribbons are also common features of confined flares. Confined events in the presence of a 
null point and with a spine-fan configuration display a circular ribbon (associated with the fan photospheric footprint) and more or less elongated remote ribbons in the regions of the spine photospheric footprint (see Masson et al. 2009, for details). In some cases, the display of more ribbons infers a more complex topology of the flaring region (e.g., Wang et al. 2014).

Eruptive flares are often associated with a flux rope. This structure, composed of flux bundles twisting around each other, is rather difficult to observe because of its low density, although prominence and filament eruptions provide the evidence that twisted structures can exist before flare triggering (Koleva et al. 2012). In some cases, the presence of sigmoids, defined by S- or inverted S-shaped coronal loops emitting in soft X-rays or EUV (Rust \& Kumar 1996), are interpreted as evidence of a flux rope buildup (Green \& Kliem 2009; Green et al. 2011; Savcheva et al. 2012b).

Recently, analyzing the Solar Dynamics Observatory (SDO) Atmospheric Imager Assembly (AIA) and the Helioseismic and Magnetic Imager (HMI) data during the 15 February 2011 X-class flare (SOL2011-02-15T01:56), Janvier et al. (2014) showed that the locations and shapes of the flare ribbons were similar to those of the intense photospheric current ribbons. These current ribbons are thin elongated structures associated with the location of high current densities, which are directly measured from the photospheric vector magnetic field observed with HMI aboard SDO. These authors also showed that during the flare evolution, the change in shape of the flare ribbons can be correlated with that of the current ribbons.

The photospheric current ribbons are interpreted as the footprints of the three-dimensional (3D) coronal current. Although the latter cannot be directly defined in observations, numerical simulations provide some insights regarding its $3 \mathrm{D}$ volumetric structure, as shown in Fig. 11 of Kliem et al. (2013). As such, the evolution of photospheric current ribbons follows that of the current layer higher up in the corona. Although this dissipation region cannot be resolved with current instrumentation, 3D numerical simulations hint of a sudden spatial collapse of the coronal current layer responsible for the sudden impulsive energy release. This was in particular studied in a series of papers, where a numerical simulation with the OHM code was set up to investigate the time evolution and underlying reconnection characteristics of a flaring erupting flux rope (Aulanier et al. 2012; Janvier et al. 2013). The photospheric footprints of the current layer are then expected to be associated with a sudden density increase following the thinning of the current layer. This sudden increase has indeed been observed in the HMI data for the first time at high spatial and temporal resolutions during an eruptive flare in Janvier et al. (2014).

Understanding the location of the energy release during a flare requires knowledge of the topology and geometry of the active region (that is, knowledge of the magnetic field line connectivity). These locations are typically associated with drastic changes of the field line connectivity, which can be discontinuous in the presence of null points and separatrices or continuous in the more generalized case of the presence of quasi-separatrix layers (QSLs; see Démoulin et al. 1996a).

Several methods have been developed over the years to identify separatrices and QSLs (see, e.g., Longcope 2005; Démoulin 2006; Janvier et al. 2015, and references within). In the presence of twisted flux tubes, an analytical study of QSLs showed that they are expected to form a $J$-shaped structure delimiting the frontier between the flux rope and the surrounding field (Démoulin et al. 1996b). The curvature of the hook section of the $J$ depends on the twist of the flux rope: it increases with an increasing twist (see Fig. 8 in Démoulin et al. 1996b). This shape was later confirmed in numerical simulations of an eruptive flux rope (Janvier et al. 2013) and in observations of eruptive sigmoids associated with a flux rope structure (Savcheva et al. 2012a, 2015, 2016).

Obtaining the QSLs associated with twisted structures in observations requires the computation of the 3D coronal magnetic field, which is performed with extrapolation methods. One of these methods consists in the extrapolation of a potential magnetic field from magnetograms, in which a flux rope structure is inserted to reproduce the coronal observational features (van Ballegooijen 2004). This approach was successfully applied to several active regions presenting sigmoids as evidence of the presence of a flux rope (Savcheva \& van Ballegooijen 2009; Savcheva et al. 2012c, 2015; Su et al. 2011). From the knowledge of the magnetic field volume, it is then possible to compute the locations of strong magnetic field distortions. Although this renders more complicated QSLs than in the analytical and numerical models, similar structures can be found, as shown in Fig. 8 in Savcheva et al. (2012a). Two-dimensional (2D) vertical cuts within the volume show the presence of an X-shaped region where one can find the highest values of the squashing degree $Q$, which defines the locations of the QSLs. This special location is referred to as the hyperbolic flux tube (HFT; Titov et al. 2002; Titov 2007; Savcheva et al. 2012a, 2016) and delineates the flux rope region within the teardrop-shaped structure above the HFT and the flare loops region underneath, as discussed in Janvier et al. (2013). In Savcheva et al. (2012c, 2015), upon studying different sigmoidal regions, the authors found that the chromospheric footprints of the QSLs obtained from the dataconstrained NLFFF models (see, e.g., Fig. 7 in Savcheva et al. 2012a and Figs. 8 and 9 in Savcheva et al. 2015) are similar to these found from numerical simulations and in observations of flare ribbons and current ribbons (see Fig. 6 in Janvier et al. 2014).

Although similarities in the shape and evolution of QSLs and the strong current density regions are expected from numerical simulations of eruptive flares (Figs. 1 and 3 in Janvier et al. 2013), no direct comparison between the photospheric footprints of the QSLs and the photospheric current densities has been made observationally for the same active region. There are several reasons for this. First of all, the ability to observe the $J$-shaped structure of the current density and its evolution from vector magnetograms requires high spatial and temporal resolutions, which were only achieved a few years ago with current instruments. Furthermore, to be able to observe fine structures in the current density maps requires $J_{z}$ levels far above the noise level (as explained in Sect. 3.1) and well-resolved magnetograms, therefore requiring active regions with strong magnetic field components that are near the disk center. Then, the number of available observations becomes limited. Comparisons with QSLs also require a good 3D magnetic field model of the studied active region, for which the structures obtained from the model match that of the observations well.

Succeeding in comparing cases for which current density structures, EUV flare ribbons, and QSLs can be all compared is paramount to assess the prediction level of numerical models, the predictions of the reconnection theory, and the understanding of the evolution of certain flare features. As such, we propose in the following to combine the analysis of the current density evolution deduced from HMI data with that of the QSL from 3D magnetic field models along with the comparison of their morphologies with that of the flare ribbons observed in the EUV band. 

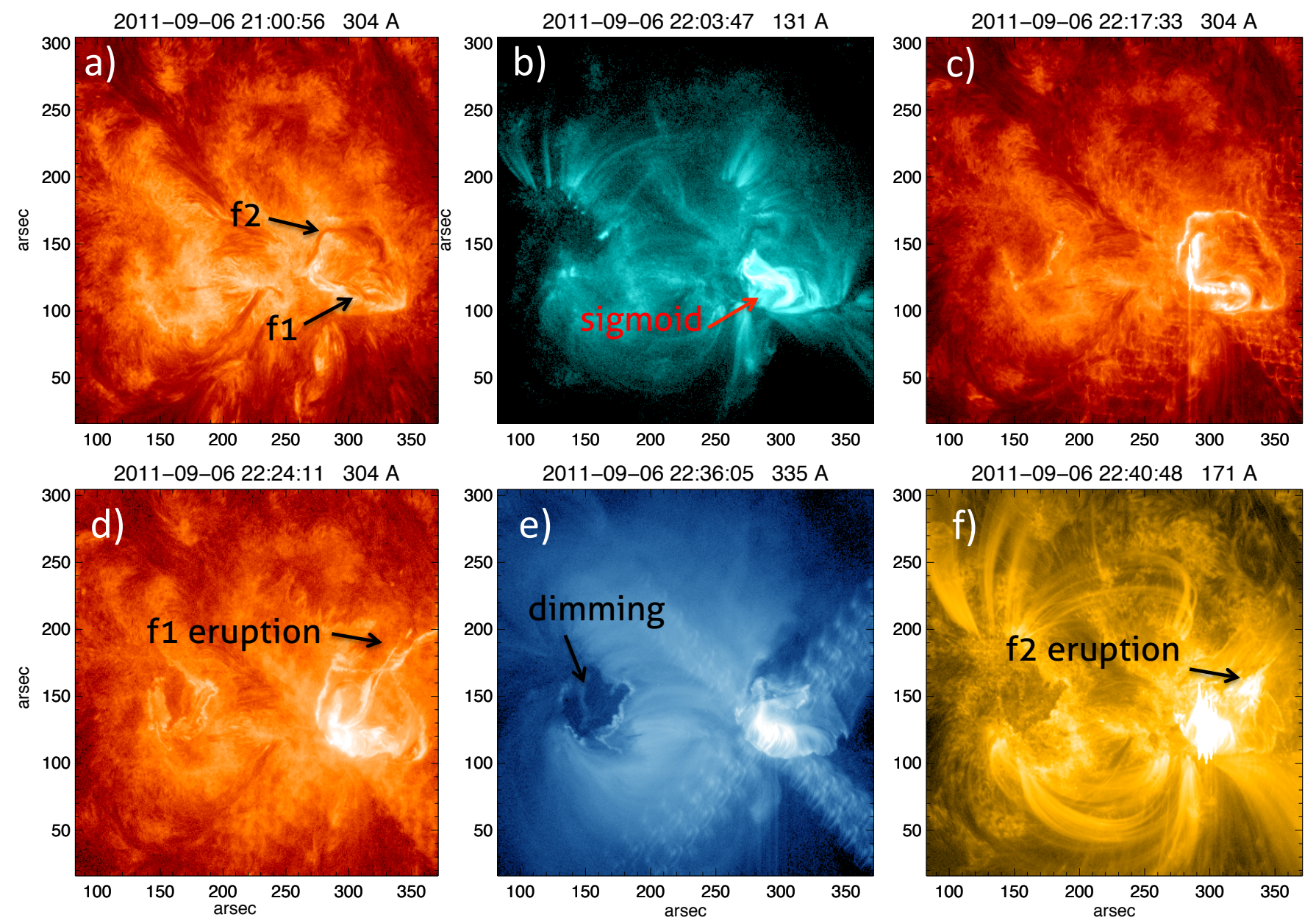

Fig. 1. a) AIA $304 \AA$ image showing the positions of the dark filaments before the flare, "f1" and "f2"; b) snapshot of the flare event SOL2011-0906T22:20 in the AIA $131 \AA$ A filter before the flare onset. In this filter, corresponding to a coronal plasma of 10 MK, an intense bright structure made of S- and/or $J$-shaped loops appears, referred to as a sigmoid, and is indicative of the presence of a flux rope. c) An image in the $304 \AA$ AIA filter, corresponding to a coronal plasma of $50000 \mathrm{~K}$, shows the flare ribbons at 22:17 UT. The ribbons first appear in the region where the brightened sigmoid is observed before the flare, while another ribbon extends in a circular shape to the north of the PIL. d) A $304 \AA$ image shows the first filament eruption (f1) that displays some twist. e) The $335 \AA$ filter shows the flare loops above the PIL and a strong dimming in the faculae region, surrounded by a faint remote flare ribbon. The flare loops appear after the onset of the flare and are mostly localized above the frontier between the strong positive polarity and negative polarity region shown in Fig. 2. f) This snapshot in AIA $171 \AA$ A shows the second, jet-like-shaped eruption of filament part f2, $16 \mathrm{~min}$ after the first eruption. An accompanying movie showing the region in different filters is available online.

The structure of the paper is as follows: Sect. 2 summarizes SDO observations of the 6 September 2011 event. Section 3 presents an analysis of the high current density regions evolving during the impulsive phase of the flare. Section 4 gives the details of the nonlinear force-free field (NLFFF) modeling. In Sect. 5, we report on the QSL computation and the comparison between QSLs and the model, the AIA flare ribbons, and the HMI current ribbons. In Sect. 6, we discuss the evolution of the unstable model and, finally, the results are summarized and we conclude in Sect. 8.

\section{The X-class flare event of 6 September 2011}

The flare that we study throughout the paper is of class X2.1 and took place on 6 September 2011 in NOAA AR 11283 (SOL2011-09-06T22:20). It occurred near disk center at position N14W18. Because of its intensity and its location, the flare has been the object of many studies (Petrie 2012; Wang et al. 2012; Feng et al. 2013; Jiang \& Feng 2013; Jiang et al. 2013, 2014a,b;
Zharkov et al. 2013; Gary et al. 2014; Liu et al. 2014; Yang et al. 2014; Xu et al. 2014). The flare, as recorded by the Geostationary Orbiting Environmental Satellites (GOES), started at 22:12 UT, peaking around 22:20 UT. Its gradual phase shows several features in the decay. However, these features are different from one EUV filter to another; for example, in the $335 \AA$ filter, another emission peak appears at around 22:40 UT. A CME was recorded by the LASCO instruments $\mathrm{C} 2$ and $\mathrm{C} 3$ with a liftoff time measured at 23:28 UT and median speed $485 \mathrm{~km} \mathrm{~s}^{-1}$. The CME evolution and energetics were studied in detail by Feng et al. (2013). Finally, the same region released another X-class flare (X1.8) a day later, at around 22:35 UT and a M6.7 flare on 8 September 2011 (Zhang et al. 2015).

The flare was recorded by AIA (Lemen et al. 2012) aboard SDO with a time cadence of $12 \mathrm{~s}$. Images at different times before, during, and after the flare are shown in wavelengths $131 \AA$, $304 \AA, 335 \AA$, and $171 \AA$ in Fig. 1. First, the intense flare peaking at 22:20 UT starts with the activation of a bright sigmoid seen in the $94 \AA$ and $131 \AA$ filters, centered at $(x, y)=\left(300^{\prime \prime}, 110^{\prime \prime}\right)$. 
The 131 A channel (panel (b)) displays well the hot plasma comprising this transient sigmoid that appears on the southeastern edge of the intrusive positive polarity around $(150 \mathrm{Mm}, 60 \mathrm{Mm})$ in the local coordinates of the magnetogram shown in the top panel of Fig. 2. This S-shaped structure is typically indicative of the presence of a flux rope (Savcheva et al. 2012b) and a filament (f1) is also observed at the same position in the $304 \AA$ filter (Fig. 1a). This filament has been studied in detail by several authors. In a series of papers, Jiang et al. used an NLFFF extrapolation and an MHD simulation to reproduce the formation of the sigmoid (Jiang et al. 2014a) and investigated the trigger process of the filament liftoff (Jiang et al. 2013). These authors concluded that the flux rope was torus-unstable prior to its eruption. Nearby, there is the filament part indicated as "f2" that does not erupt at the same time as the former region. Finally, another filament further away to the west from the present region was found (Jiang et al. 2014b), but remains stable over the course of the flare and, as such, does not play a role during the flare.

The $304 \AA$ images (panels (a), (c), and (d)) show well the filament material before the eruption (panel a) and during (panel (d)) as well as the flare ribbons with no saturation (panel (c)). The two filamentary parts $\mathrm{f} 1$ and $\mathrm{f} 2$ in panel (a) are associated with two eruptions occurring at $16 \mathrm{mn}$ interval (panels (d) and (f)). The $335 \AA$ channel (panel (e)) shows the flare arcade with very little saturation well and also a strong dimming appearing on the east side of the active region, at $\left(150^{\prime \prime}\right.$, $\left.150^{\prime \prime}\right)$, corresponding to the faculae region appearing in the magnetogram of Fig. 2.

The sequence of events continues at 22:10 UT with the appearance of kernel brightnings that are also captured in the visible continuum ( $\mathrm{Xu}$ et al. 2014). These kernels are strong emission patches at chromospheric and photospheric levels and evolve into two ribbons at 22:14 UT. They quickly grow to their full length at 22:18 UT. Contrary to the typical two-ribbon flare morphology (such as in Chandra et al. 2009), the present two ribbons begin almost perpendicular to each other. As the two ribbons evolve, a circular ribbon lights up, encircling the tworibbon structure and accompanied by a remote ribbon in the faculae region centered at $(x, y)=\left(150^{\prime \prime}, 150^{\prime \prime}\right)$ surrounding the remote dimming; see panels (c)-(e).

These four ribbons suggest the presence of two systems: a two-ribbon system associated with the flux rope and filament f1, possibly elongating to the $\mathrm{f} 2$ filamentary part, and another system associated with the presence of a magnetic null. The circular ribbon is interpreted as the chromospheric signature of the fan dome. The remote ribbon in the faculae may be a signature of the spine; the elongation of the spine ribbon was explained by Masson et al. (2009) for a null configuration embedded in a quasi-separatrix layer. Such a structure can be suggested with a look at the magnetogram (Fig. 2), where a parasite positive polarity is surrounded by a region of negative polarity. Magnetic field extrapolations can be performed to confirm these hypotheses, as is shown in more detail in Sect. 4.

Finally, at 22:24 UT, the filament $\mathrm{f1}$ material escapes upward while the emission saturates in most of the AIA filters. This is followed by the formation of hot loops over the polarity inversion line (PIL). Then, at 22:39 UT, the eruption of filament $\mathrm{f} 2$ is seen erupting in absorption, in the $171 \AA$ filter which shows the jet-like ejection in good detail (panel f), while in the meantime hot loops appear connecting the parasite polarity edge and faculae region.
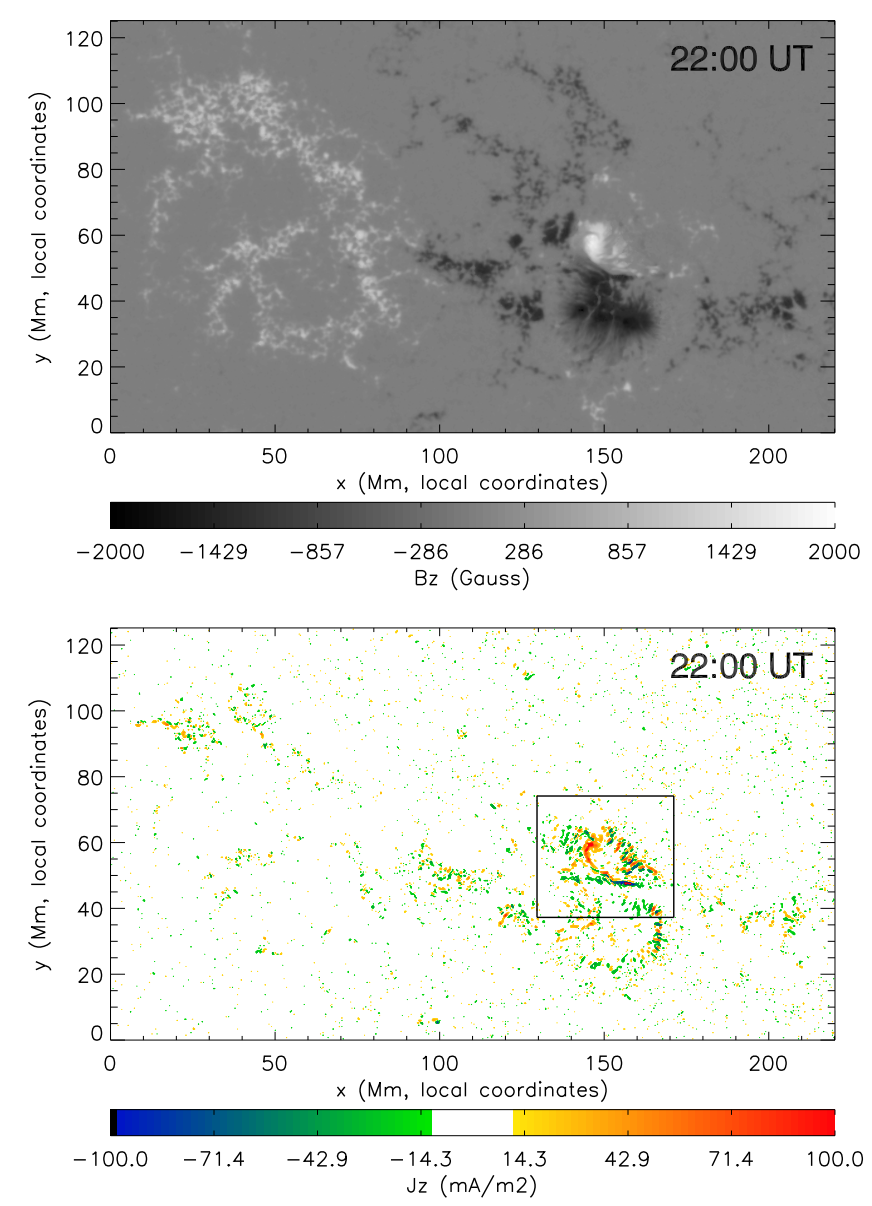

Fig. 2. Top: HMI magnetogram of the active region 11283 before the flare, taken at 22:00 UT, showing a leading bipole made of a small strong positive polarity and more extended negative polarity and a trailing faculae region of positive polarity. Bottom: map of the same field of view, obtained at the same time, of the vertical component of the current density, $J_{z}$, with the noise level removed. The leading strong bipole shown in the magnetogram corresponds to the location of the strongest current. The square indicates the field of view where the evolution of the currents and QSLs are investigated in more detail (see Fig. 3).

\section{Evolution of the electric current in the $J_{z}$-ribbons}

\section{1. $B_{z}$ and $J_{z}$ maps}

The flare was also recorded with HMI (Schou et al. 2012; Scherrer et al. 2012), which provided photospheric vector magnetic field measurements of the region every $12 \mathrm{~min}$. The magnetogram presented in Fig. 2 is a map of the vertical component of the magnetic field $B_{z}$ at 22:00 UT, before the flare occurrence. To obtain this map, we inverted the HMI level-1b IQUV data in the region covering AR 11283 with the Milne-Eddington inversion code UNNOFIT (for details see Bommier et al. 2007; Janvier et al. 2014). In particular, we chose this method because of its good determination of the field inclination. This inversion was made on a set of 15 maps, from 2011/09/06 21:00 UT to 2011/09/07 00:00 UT, while applying a solar rotation compensation to select the same region over these three hours of observation. The $180^{\circ}$ azimuth ambiguity remaining after the inversion was resolved by applying the ME0 code developed by Leka et al. (2009) and, finally, the magnetic field vectors were rotated into the local reference frame, also referred to as the heliospheric reference frame, where the local vertical axis is the $O z$ axis and the 
photosphere lies in the $O x y$ plane. The mesh size of the map is $\approx 385 \mathrm{~km}$ in the $x$-direction and $\approx 370 \mathrm{~km}$ in the $y$-direction.

The magnetogram from Fig. 2 shows a large decaying active region extending over $200 \mathrm{Mm}$. The leading part of the active region is composed of a strong negative polarity region and a small parasitic positive polarity region of strong magnetic field strength (values of $\left|B_{z}\right|$ of around $2000 \mathrm{G}$ ). The negative polarity region shows some fragmentation and is followed by a trailing, faculae region with values of the photospheric magnetic field well below $2000 \mathrm{G}$. Both sigmoid and filaments discussed in Fig. 1 are localized above the PIL between the parasitic positive polarity and extended negative polarity, centered at $(x, y)=(150 \mathrm{Mm}, 50 \mathrm{Mm})$ in the local coordinates of Fig. 2.

In the bottom panel of Fig. 2, we also present a map of the vertical component of the current density, $J_{z}$ at 22:00 UT. This map, shown in the local solar coordinates, has the same field of view as the magnetogram. The current density is directly derived from the magnetic field components obtained with the inversion method aforementioned via a centered difference method from the equation, $\nabla \times \boldsymbol{B}=\mu_{0} \boldsymbol{J}$, written in the local frame. We investigated both the evolution of the perpendicular and parallel components (relative to the vertical magnetic field component $B_{z}$ ) of the current density. However, since $J_{\perp}$ does not evolve much during the flare, we focus in the following on the evolution of its vertical component $J_{z}$. Looking at the evolution of this component is of high interest for extrapolations of the solar magnetic field (as discussed, e.g., in Wheatland \& Gilchrist 2013). Finally, we removed the $J_{z}$ components below the estimated noise level ( $20 \mathrm{~mA} \mathrm{~m}^{-2}$; see Gary \& Démoulin 1995) in every map shown in the following, which corresponds to white areas in Fig. 2.

The region of interest where the most intense emissions during the flare take place is rather small compared to the whole active region; this region is indicated with a square in Fig. 2 (bottom) and corresponds to the area where the intense flare ribbons are also seen, as shown in Fig. 1 in the $304 \AA$ filter before (a) and after the flare peak (c) and the location of the sigmoid (panel b). As such, even though the active region covers a large area (over $200 \times 120 \mathrm{Mm}^{2}$ ), the flare itself occurs in this small region corresponding to a bipole made of a strong positive and negative polarity. This region also has the strongest electric current densities with elongated shapes along the PIL. Previous authors also reported on the change in the horizontal magnetic field (Petrie 2012; Liu et al. 2014). While they mainly focused on the Lorentz force, in the following we interpret the changes in the electric currents in light of the 3D standard flare model.

The positive (resp. negative) density currents (in red, resp. in green) mostly appear in the positive (resp. negative) polarity near the inversion line, but the tendency changes further away from the PIL, where "salt and pepper" structures are found. This behavior can be explained by the presence of the interlocking-comb structure in the penumbra of the two sunspots (Thomas \& Weiss 2012; Solanki \& Montavon 1993) as can be seen by comparing both $B_{z}$ and $J_{z}$ maps. Indeed, the variation of optical opacity across the fibrils, which points to a magnetic field that is not fixed to the same height, participates in the modulation of the currents in the penumbra, therefore showing a zebra pattern (Gosain et al. 2014). Further descriptions of the structures seen in the region of interest are given below.

\subsection{Evolution of electric current ribbons}

The evolution of the vertical component of the current density $J_{z}$ is presented in Fig. 3 four times, two before and two after the impulsive phase of the X-class flare (left column). The colors in the yellow-red range indicate positive current densities, while colors in the green-blue range indicate their negative values, similar to Fig. 2. As described earlier, most of the positive current density is found in the positive magnetic polarity, and inversely, the negative current density is found in the negative magnetic polarity. Both positive and negative current densities show elongated structures along the PIL, especially before the flare occurrence at 22:00 and 22:12 UT, suggestive of opposite current ribbons. The positive current density ribbon exhibits a hook shape in its northeast tip that is similar to the current ribbons found in the 15 February 2011 event (see Janvier et al. 2014). However, the negative current density ribbon only shows a straight part and no hook structure. This may be due to the complexity of the bipolar region, which was pointed out in the description of the flare ribbons; these ribbons do not exhibit the typical two hooked shapes that would usually be found in an eruptive flare.

We also show four marked regions (boxes 1-4) superimposed on these $J_{z}$ maps that highlight areas of strong changes in the current density during the flare. All these regions focus on different areas of the current density ribbons. Before the flare peak (at 22:00 and 22:12 UT), these boxes are associated with low or extremely low values of the current density. Only region 2 is associated with small patches of both positive and negative currents. As the flare occurs, the current density increases in all of these boxes (see 22:24 and 22:36 UT). This increase is accompanied by a spreading of the current ribbons in the west direction in box 1 , in the north direction in box 2 , and in the south direction for boxes 3 and 4 . Around the end of the flare (22:36 UT), these areas are filled with high values of positive and negative current density that persisted long after the peak of the flare. In box 2, where we found both positive and negative current density before the flare, the patches of positive $J_{z}$ have grown and amalgamated to form two big patches of positive current only.

The right column of Fig. 3 shows maps before and after the flare for the same times, but displaying base-difference images with a base image chosen long before the flare at 21:36 UT. The base-difference images therefore show the evolution of the difference between the current density at the time considered and 21:36 UT. Red indicates an increase in the current density compared with the levels obtained at the base time, while blue indicates a decrease in the current density. We only consider the evolution of the direct currents, i.e., the values of $J_{z}$ that satisfy $J_{z} \cdot B_{z}>0$ in both magnetic polarities. Similar to the $J_{z}$ maps, different areas are highlighted in the base difference images. In each of these boxes, one can see strong changes in the direct current densities (shade of red) just after the start of the impulsive phase (22:24 and 22:36 UT). Especially, as pointed out earlier, the ribbons spread outward from the PIL (west-north direction in the positive magnetic polarity and south direction in the negative magnetic polarity) with the area indicated by the red box 1 showing the strongest increase.

\subsection{Electric current increase in the impulsive phase}

Taking the different regions highlighting the areas with the strongest changes (boxes 1-4 in Fig. 3), we can integrate the current densities over their related surface to get the electric current values $\left(I=\int_{S} J \mathrm{~d} S\right)$. This can be carried out for direct currents (with the condition $J_{z} \cdot B_{z}>0$ ) or return currents $\left(J_{z} \cdot B_{z}<0\right)$, therefore separating both contributions in each of the regions considered. 

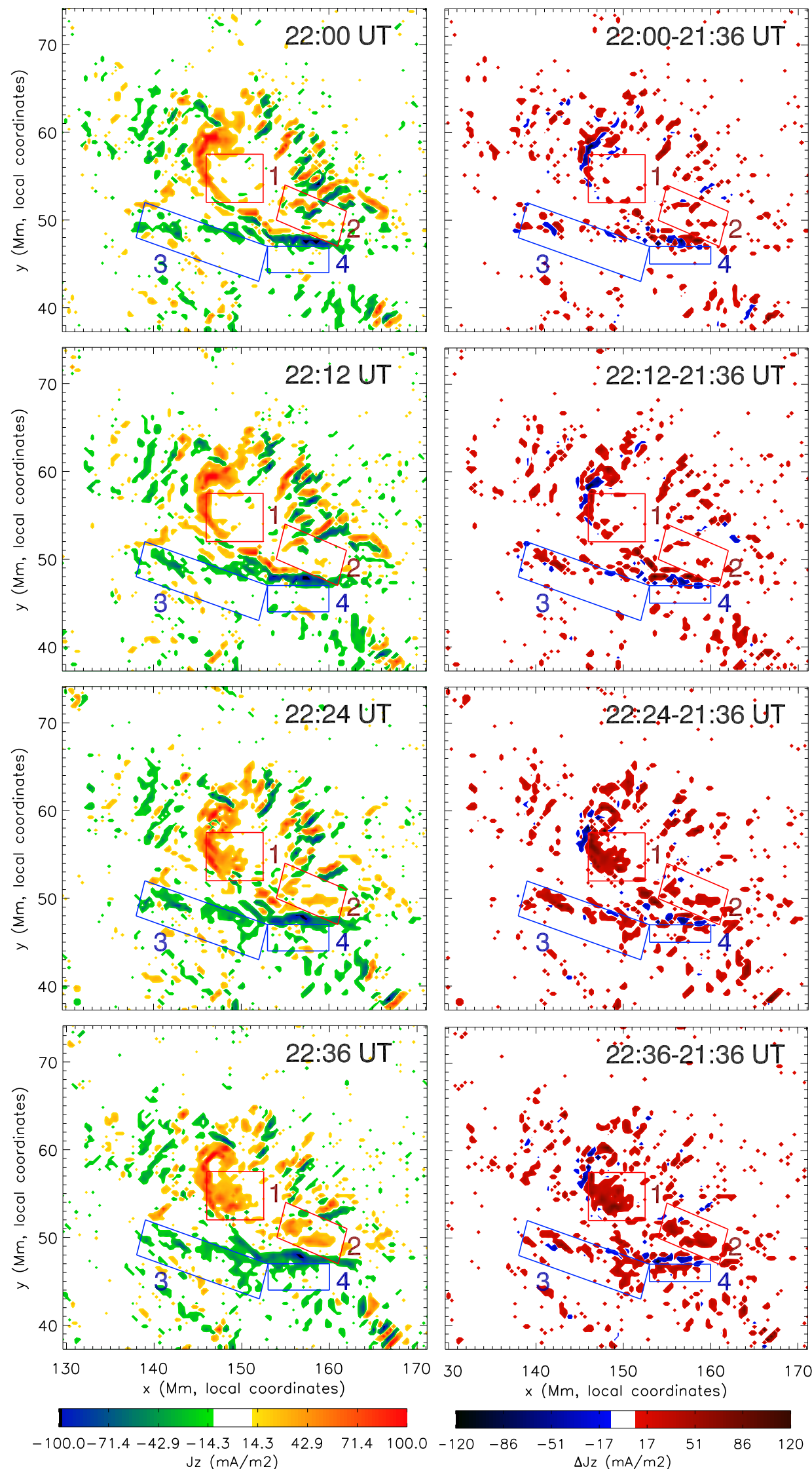

Fig. 3. Left: $J_{z}$-maps of the zoomed portion of the active region (see Fig. 2) two times before (22:00 and 22:12 UT) and after (22:24 and 22:35 UT) the flare in the solar local coordinates. Right: base-difference of the direct current density, where the base is taken at 21:36 UT, the red (resp. blue) color showing an increase (resp. decrease) in the density. The four boxes indicate the regions where most of the changes before and after the flare are seen and where the electric current is integrated. Two boxes ( 1 and 2 ) are chosen in the positive magnetic polarity, while two others ( 3 and 4$)$ are chosen in the negative magnetic polarity to point to the most important changes during the flare evolution. 

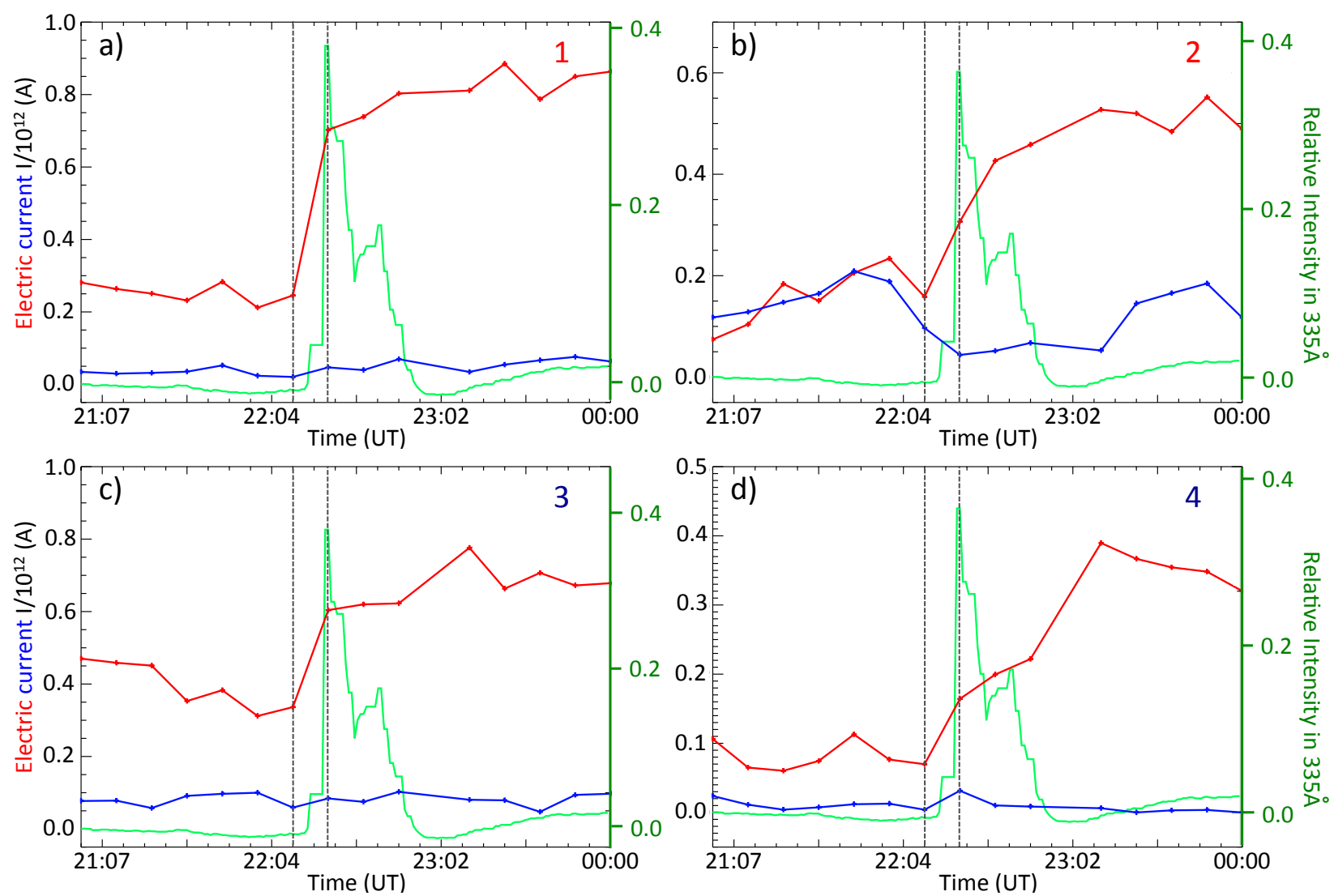

Fig. 4. Evolution of the direct electric current (in red) and the return electric current (in blue) before, during, and after the X-class flare in regions 1 a), 2 b), 3 c), and 4 d). The light curve (relative intensity normalized to the intensity at 21:00 UT) is also added, corresponding to the full Sun signal in the SDO $335 \AA$ A channel (green). The beginning and end of the flare impulsive phase are indicated with dotted vertical lines.

The time evolution of the current intensity $I$ is shown in Fig. 4. For each plot, we reported the values of the direct and return electric currents (respectively in red and blue) and the relative intensity of the flare in the $335 \AA$ SDO channel (green plots), so as to show the impulsive phase of the flare. In each region, the direct current is shown to increase during the impulsive phase of the flare. The increase is about 2.8 times in box 1 , 1.9 times in box 2, 1.8 in box 3 , and finally 2.4 in box 4 .

On average, the electric current increase is about two times the value before the impulsive phase of the flare. This value is similar to that found in the X-class flare event of 15 February 2011 (Janvier et al. 2014), for which the authors had pointed out for the first time the evolution of the current ribbons with a similar morphology as the present case. In the February 2011 flare, the electric current density also increases in some regions of the current ribbons, namely near the straight part and at the tip of the hook of the $J$-shaped ribbon and the current ribbons display a very clear $J$ shape. The locations of current increase were also associated with strong hard X-ray emissions (Musset et al. 2015).

\section{NLFFF modeling}

As our aim is to place the current ribbons in topological context and to show how they coincide with the flux-rope associated QSLs (as suggested in Aulanier et al. 2012; Janvier et al. 2013, 2014), we need to obtain a detailed model of the 3D magnetic field structure of the region. Since the region contains clear S-shaped loops over part of the PIL (see Fig. 1), we resort to building NLFFF models, which can best represent a region with a strong torsion parameter in the core, surrounded by an envelope of weak- $\alpha$ region in the overlying, more potential arcade. In the following, we use the flux rope insertion method to produce 3D magnetic NLFFF models for this region. The description of the method is given in detail in Savcheva \& van Ballegooijen (2009), Su et al. (2011), and Savcheva et al. (2012b). In a nutshell, the method consists in the following steps: 1) perform a global potential field extrapolation from a SOLIS synoptic magnetogram; 2) select a high resolution region from the HMI magnetogram and perform modified potential field extrapolation with side boundary conditions given by the global field; 3 ) insert a flux rope along the path of an AIA $304 \AA$ filament as a combination of axial and poloidal fluxes; 4) relax the configuration to force-free equilibrium by magnetofriction (MF); and 5) build a grid of models with different flux rope paths and combinations of axial and poloidal fluxes. The grid covers poloidal fluxes in the range $\left[10^{9} \mathrm{Mx} \mathrm{cm}^{-1}, 1.5 \times 10^{10} \mathrm{Mx} \mathrm{cm}^{-1}\right]$ and axial flux in the range $\left[10^{20} \mathrm{Mx}, 5 \times 10^{20} \mathrm{Mx}\right] ;$ 6) Match each model to observed coronal loops from the X-ray Telescope on Hinode (XRT; Golub et al. 2007) and AIA and issue a best-fit, stable preflare model. The criteria for selecting the best-fit model is described in detail in Savcheva \& van Ballegooijen (2009). It relies on calculating the minimal average distance between segments from field lines traced from the models projected onto the disk and segments of loops traced from XRT or AIA that underlay these field lines along the line of sight. The different choices for the flux rope path leading to the best-fit model are presented in more detail in Appendix A.

Sample field lines from the preflare best-fit and stable model, referred to in the following as model S, are shown in Fig. 5. We 


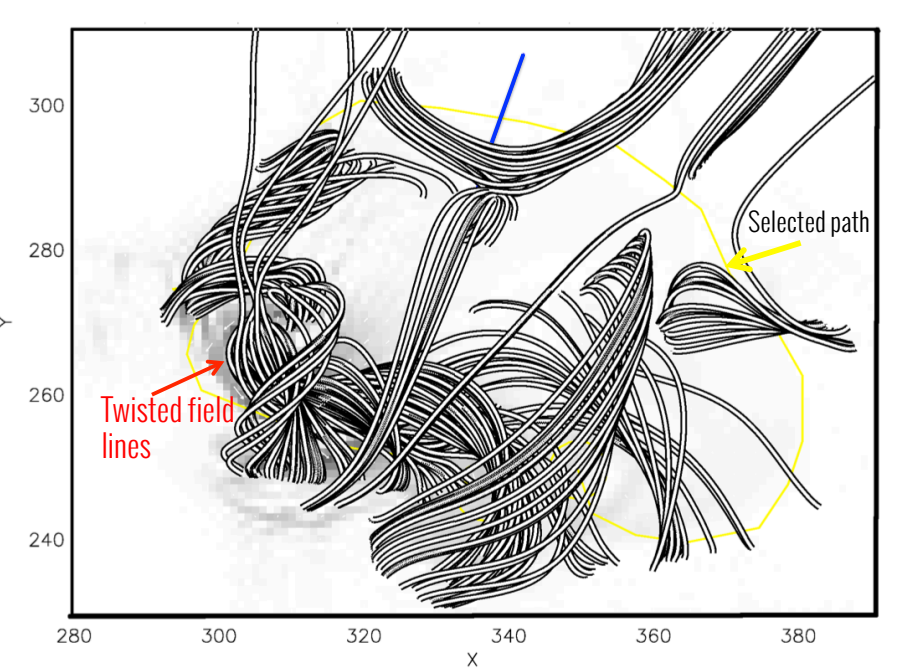

Fig. 5. Randomly chosen field lines representing the best-fit model (model S, see right panel in Fig. A.1) after relaxation. The $x$ and $y$ coordinates are in model coordinates viewed from top down on the region. Some of the field lines are sheared and some are twisted, mostly along the PIL of the parasitic positive polarity and the negative leading polarity of the active region. Away from this region, the field lines are mostly low-lying arcade loops, while the northern region has open field lines surrounding the HFT region (Sect. 4).

used the S-shaped field lines that can be seen in panel (b) of Fig. 1 along with the corresponding image in XRT to match the observations and models. The poloidal and axial fluxes of model $\mathrm{S}$ are $10^{9} \mathrm{Mx} \mathrm{cm}^{-1}$ and $4 \times 10^{20} \mathrm{Mx}$. This model displays twisted field lines only over some portion of the southeastern part of the PIL (some of these are indicated with a red arrow in Fig. 5) at the same location as the sigmoid in the observations. These twisted field lines dip under some sheared arcade field lines and then emerge on the other side. Although the best-fit flux rope path corresponds to the best match with the circular filament in Fig. 1a, after the MF relaxation most of the shear and twist over the rest of the PIL are dissipated and a flux rope only remains where the double ribbons are seen. This provides strong evidence that the model does not necessarily output a flux rope everywhere where a flux rope is inserted as an initial condition, i.e., the ambient magnetic field structures play a vital role in determining the final relaxed 3D NLFFF magnetic field.

The rest of the PIL shows arcade loops over the PIL and "open" field lines. Because of the nature of the method we naturally obtain that most of the active region is potential, which can be seen from a comparison with a potential field extrapolation from the same magnetogram. However, this is confirmed by a model of the same active region at the same time produced with the quasi-Grad-Rubin method for NLFFF models from line-of-sight magnetograms, which by no means requires potentiality anywhere in the active region (for details see Malanushenko \& Savcheva 2016). This is dissimilar to the previous extrapolations (Feng et al. 2013; Jiang et al. 2013; Liu et al. 2014), which only showed a confined flux rope underneath arcade loops. At the same time, the present configuration with the presence of a flux rope provides evidence that a flux rope can be embedded in a complex topology, as was reported in other events, for example, in the X-class flare of 23 October 2012 (Yang et al. 2015) and that of 29 March 2014 (Liu et al. 2015).

As described in Savcheva et al. (2015, 2016), we can also produce unstable flux rope models to replicate the flaring configuration. This stability analysis was first introduced by

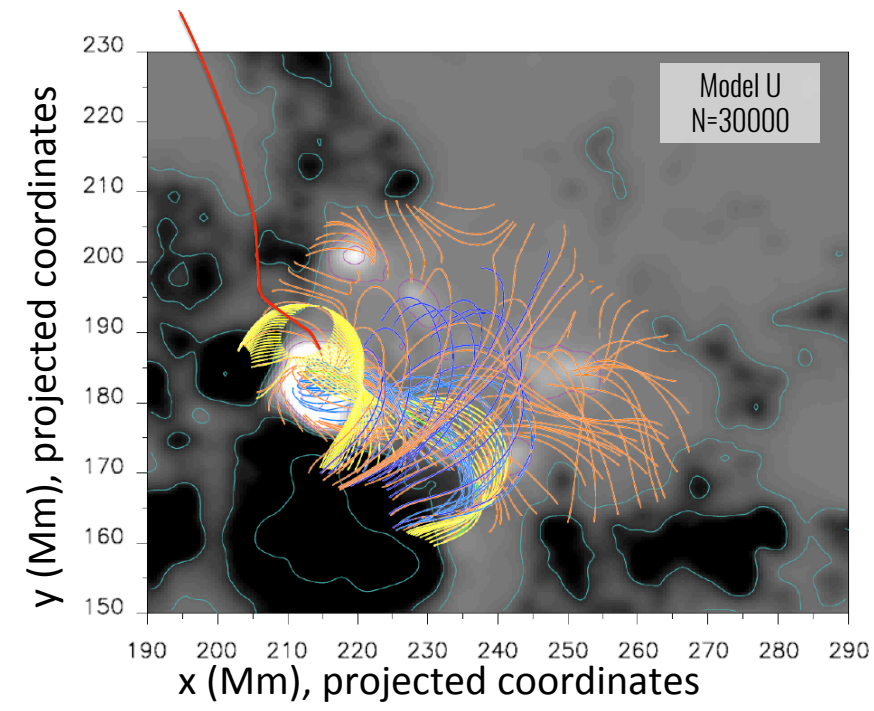

Fig. 6. Different sets of colored field lines representing model $U$ at iteration time $n=30000$. The yellow lines represent closed field lines situated under the fan dome, and associated with the flux rope, while the blue and orange lines are more potential field lines also situated under the dome. The red line represents the spine that departs from the null point found in model $\mathrm{U}$.

Su et al. (2011) for the magnetofrictional simulations with the flux rope insertion method and then confirmed in full MHD by Kliem et al. (2013). These unstable models are produced by adding axial flux to the best-fit stable or marginally stable flux rope (here model $\mathrm{S}$ ) to push it over the edge of stability. This effect of the axial flux, rather than the poloidal flux, has been discussed in detail in Su et al. (2011). As shown in Fig. 10 in Savcheva et al. (2016) the effect of the increased axial flux is the constant elevation of the flux rope with continued iteration, which may never stop and could result in an erupting flux rope or a new higher equilibrium (in principle, this is possible, but found only in one case in Kliem et al. 2013). In this sense, adding more axial flux just increases the residual Lorentz force that pushes the flux rope out of equilibrium and causes it to rise faster, although the magnetofrictional evolution of the flux rope with more or less axial flux seems to be self-similar.

The unstable model that we use here to represent the flaring configuration is referred to as model $U$ in the following, and has an axial flux of $5 \times 10^{20} \mathrm{Mx}$ and the same poloidal flux as model S. As was shown extensively in Savcheva et al. (2016), the different iterations of the magnetofrictional relaxation evolve the configuration in a similar way as a fully dynamical MHD simulation. model $U$ is shown in Fig. 6 at an early iteration $(n=30000)$, where different field lines were selected to represent the different topological regions. Since this model is associated with the existence of a null point (NP), the fan structure is shown with yellow and blue field lines situated under the dome, while a red line represents the spine. The yellow lines are also chosen to represent twisted field lines associated with the flux rope, which is found at a similar location as model $\mathrm{S}$, over the southern part of the PIL.

Further iterations of model $\mathrm{U}$ show that the flux rope expands and stretches in the vertical direction. This evolution can be seen from Fig. 7, where we show a cross section of the current density volume through the middle of the flux rope. This cross section is shown for the stable preflare model (model S, panel a) and the different iterations of the unstable model (model $\mathrm{U}$, panels $\mathrm{b}-$ d). As can be seen from all panels, the current layers exhibit 

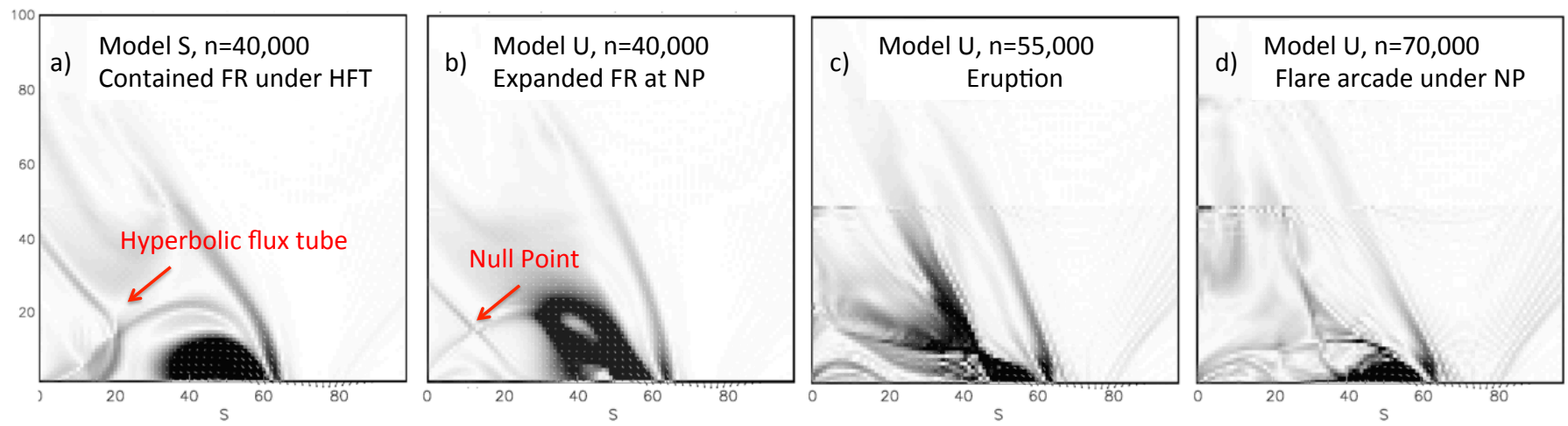

Fig. 7. Vertical cross section of the currrent density volume. The cross section, indicated with the blue line in Fig. 5, is shown for a) the stable model S, at iteration step 40000 , and then for model $U$ at different iteration steps b)-d). a) The X-crossing of some current structures indicates the presence of an HFT. For the unstable model U, the traces of the flux rope, surrounded by the thick current layer area, can be seen as it lifts up at iteration $n=40000 \mathbf{b}$ ). At that point, the flux rope passes through the null point at $n=55000 \mathbf{c}$ ) and disappears, leaving coronal loop arcades underneath the null point $\mathrm{X}$-structure at $n=70000 \mathbf{d}$ ).

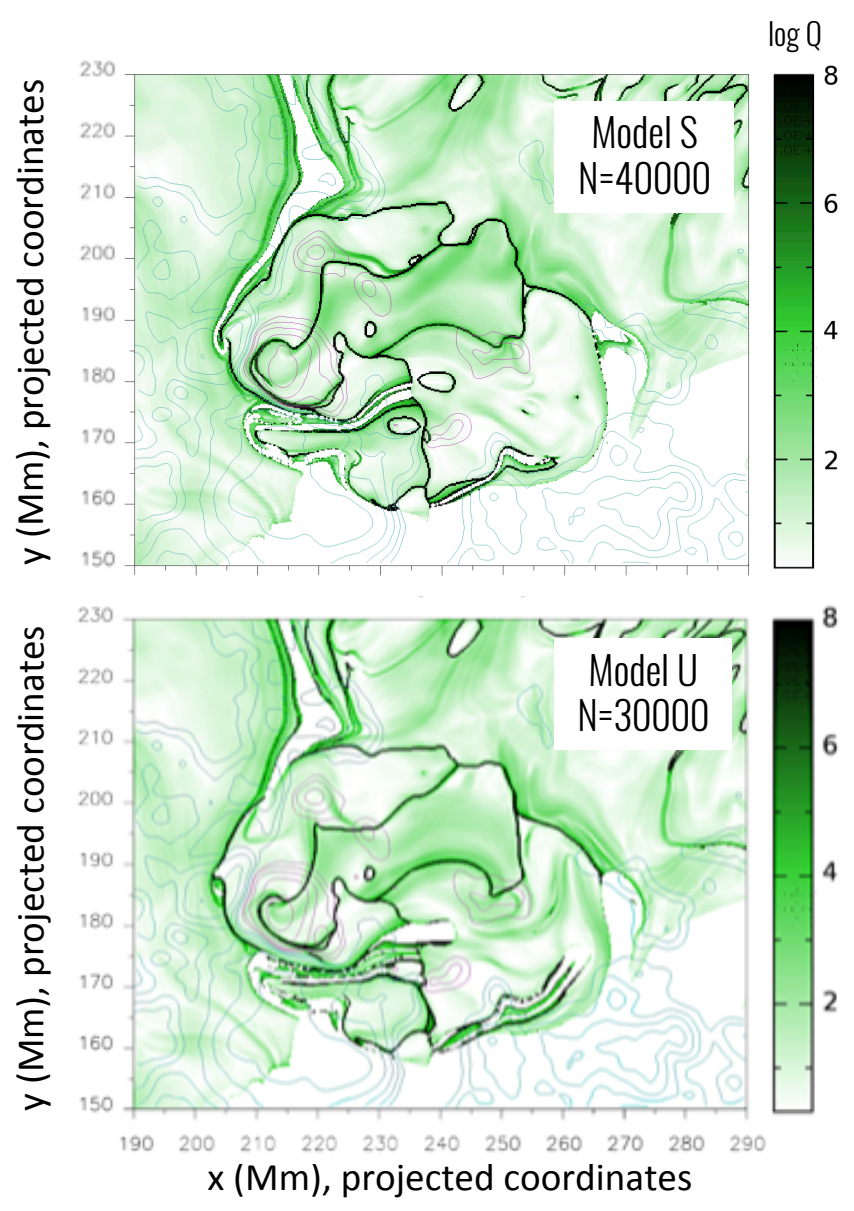

Fig. 8. QSL maps at a height of $4 \mathrm{Mm}$ for the stable model (top) and unstable model (bottom). The high squashing factors are shown in dark green, while the squashing factor computed on open field lines (in the numerical domain) is shown in white. The global geometry (circular shape) of the QSLs are similar for the two models.

a crossing shape (most notably seen above the flux rope for model S), which we associate with the location of the highest squashing factor values or a hyperbolic flux tube, i.e., where the magnetic field is the mostly distorted and found at similar locations as the high electric current densities (see, e.g., Janvier et al. 2013). The same location corresponds to a NP that we found for model $\mathrm{U}$ ( $n=30000$, as indicated in (c)). While the flux rope is evolving, it pushes against the NP and the current at the edge of the flux rope intensifies (b). The flux rope is accompanied by an HFT underneath, where reconnection is most likely to take place. This is similar to the study by Zhao et al. (2014), where the authors found a small narrow flux rope in the famous AR 11158 with an HFT underneath and above just before the X-class flare. The motion of the flux rope upward compresses the current sheet at the NP, where reconnection ultimately allows the flux rope to escape (c-d). This dynamic, inferred from the snapshots of the magnetofrictional simulation, can be confirmed by a MHD simulation with a similar topological configuration. We leave this confirmation for a future study achieved with the method developed in Kliem et al. (2013). In panel (d), the flux rope has already escaped and a flare arcade of more potential fields has formed within the dome under the NP.

\section{QSLs, flare ribbons, and current ribbons}

\subsection{QSL computation}

The pioneering work of Priest \& Démoulin (1995) and Démoulin et al. (1996a) showed that the gradient of the field line mapping from one set of footpoints to the other can be used to quantify the change in linkage. In particular, an invariant quantity called the squashing factor, $Q$, was proposed to calculate the deformation of the magnetic field in a volume (Titov \& Démoulin 1999; Titov et al. 2002; Titov 2007). Regions of high squashing factor are called quasi-separatrix layers (QSLs), and can be computed from the magnetic field data cube derived from the magnetic field models (see, e.g., Savcheva et al. 2015, 2016).

The QSL maps shown in Fig. 8 are computed employing the iterative method of Pariat \& Démoulin (2012) where an adaptive mesh is defined based on the value of $Q$ and the refinement is carried out by increasing the resolution at each refinement step until a set convergence limit is reached. This method has been widely used to compute the squashing factor in numerical simulations (e.g., Aulanier et al. 2006; Janvier et al. 2013) and recently to compute the QSLs to provide 2D maps of the connectivity from magnetic field models (see, e.g., Savcheva et al. 2012a, 2015, 2016). As in other studies (e.g., Savcheva et al. 2012a; Zhao et al. 2016), the reference boundary for the QSL computation is chosen slightly above the photosphere, here at $z=4 \mathrm{Mm}$, 


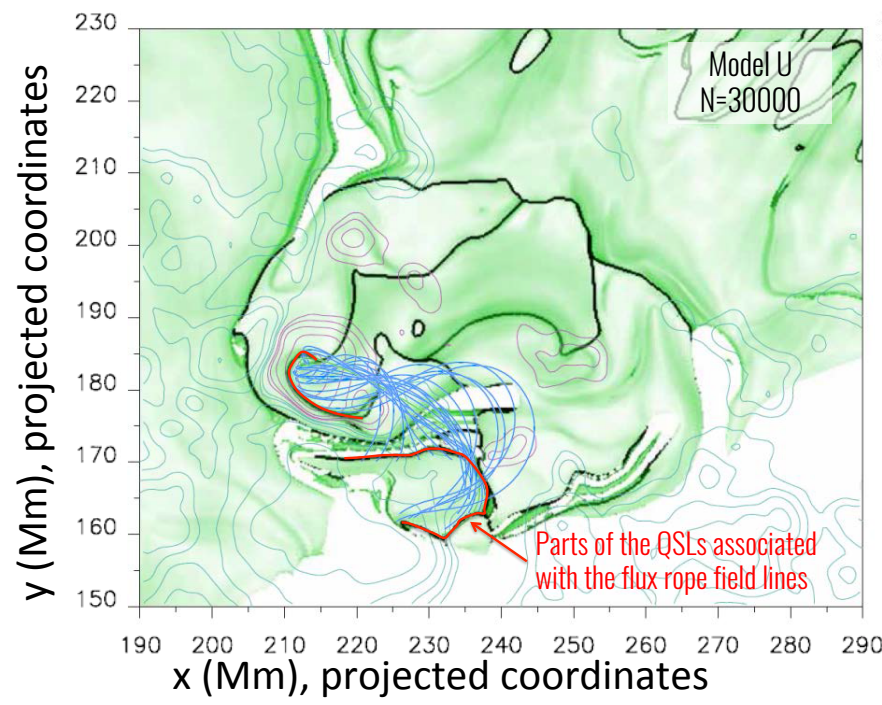

Fig. 9. QSL map for model $\mathrm{U}$ at iteration time $n=30000$ showing selected field lines in blue representing the flux rope (see Fig. 6). Their footpoint mapping onto the photosphere corresponds to a certain portion of the QSLs of the entire region.

to solely focus on the important large-scale topological structures and to exclude QSLs related to small-scale polarities at the photosphere. In Fig. 8, the QSL photospheric mapping is shown for the stable and unstable flux rope models (models $\mathrm{S}$ and $\mathrm{U}$ ). Both cases display a large-scale, dark high- $Q$ area that surrounds the parasitic positive polarity and also coincides with the footprint of the NP fan with the photospheric surface (model U). Inside this dark surrounding QSL the high- $Q$ regions are highly structured, especially where the magnetic field reverses around $(210<x<240,160<y<180)$. It is important to note that there are white regions in the maps where the squashing factor could not be computed because some field lines are leaving the computational domain. Although the location of the white regions and their shape depend on the size and extent of the computational domain, choosing a given computational domain can be useful to delineate different prominent topological features. For example, we see that there are white "open" regions intertwined with the flux rope QSLs. These regions provide an escape route for flux rope field lines to leave the fan dome in a direction different from that of the spine (we discuss this further in Sect. 6.2).

In Fig. 9, the same QSL map for model $\mathrm{U}$ is presented, overlaid with a few blue magnetic field lines. These field lines were selected so as to represent the flux rope within the NP dome. Their footpoints are anchored on the photospheric surface, where we find the southernmost QSLs. We indicated their specific locations with red curves. Interestingly, these latter show a $J$ shape as expected from previous studies on the QSLs for a flux rope (Savcheva et al. 2012a; Janvier et al. 2013). Indeed, the presence of twisted field lines is typically associated with QSLs. Their photospheric traces encircle the endpoints of the flux rope field lines. The degree of winding (whether the hook of the QSLs is wrapping onto itself) is related to the degree of twist of the flux rope (see, e.g., Fig. 8 in Démoulin et al. 1996b). Therefore, even though the overall topology of the present active region is complex, with the presence of a null point and associated features (fan and spine), we were able to find the typical shape of flux rope-associated QSLs. In the following, we compare this shape with the flare ribbons observed with AIA.

\subsection{Flare ribbons and QSLS}

In Fig. 10, we show a large- and small-scale field of view of the flare ribbons obtained in different filters. We chose the $304 \AA$ filter because it displays well the large-scale structure of the flare ribbons, although the ribbons associated with the erupting filament $\mathrm{f} 1$ become saturated closer to the peak: we then chose the $1600 \AA$ filter in this zoomed region, as the ribbons saturate later in this filter. We overlaid the traces of the QSLs for the same field of views on the AIA observations. This technique, which compares the images of the ribbons and the 3D QSLs was first presented in Savcheva et al. (2015). The different times in this figure are chosen to best match the EUV flare ribbon observations. We selected times well before the peak of the flare for a comparison with the stable model (S) at 22:03 UT. For the unstable model (U), the comparison works the best for an observation time taken near the peak of the flare, at 22:14 UT for the filament region (the ribbons become too saturated nearing the flare peak), and at 22:17 UT for the circular ribbon.

At this time, the circular ribbon of the fan appears clearly, especially during the flare (panel b). The circular outer ribbon is overall reproduced well by the large-scale QSL map, although the west and south part of the circular ribbon $\left(320^{\prime \prime}<x<\right.$ $\left.350^{\prime \prime}, 100^{\prime \prime}<y<180^{\prime \prime}\right)$ is reproduced only by lower- $Q$ QSLs, which are not shown in the figure. Another structure that matches well is that of the flare ribbon branch extending from $(x=$ $\left.285^{\prime \prime}, 130^{\prime \prime}<y<160^{\prime \prime}\right)$; we also find a QSL branch extending in the same locations.

The region where the filament $\mathrm{f} 1$ is found (between $(x, y)=$ $\left(280^{\prime \prime}, 120^{\prime \prime}\right)$ and $(x, y)=\left(320^{\prime \prime}, 120^{\prime \prime}\right)$, see Fig. 1a) corresponds to the region where we found the $J$-shaped flux-rope QSLs (Fig. 9). Although the matching is not perfect (panels c and d), there are several similarities with the flare ribbons, such as the elongated central sections and hook of the flare ribbons. Such a resemblance can be found in other flux rope eruptions in a complex topology (embedded in a null point dome), as was reported by Yang et al. (2015; see their Fig. 8). The motion of the ribbons away from each other are also reproduced well by the QSLs during the flare (panel d at 22:14 UT). Those common structures are indicated with red arrows. Since model $S$ is the stable flux rope case while model $U$ is the unstable case, this is a nice example of the flux rope insertion method working well for different phases of the flare.

\subsection{QSLs, flare ribbons, and current ribbons}

The shape of the flare ribbons and QSLs and their evolutions, can also be compared with the current ribbons described in Sect. 3. While Fig. 3 (left panels) showed the evolution of the electric current densities two snapshots before and after the peak of the flare, in Fig. 11 we show an overlay of these high density areas on top of the $1600 \AA$ flare ribbons obtained with AIA.

In panels (a) and (d) of Fig. 11, we show the flare ribbons at 22:03 UT (a) and 22:14 UT(b). At 22:03 UT, the ribbons are already formed well and display the typical $J$-shaped, tworibbon structure associated with filament eruptions (see, e.g., Chandra et al. 2009). Compared with the time 22:03 UT, the flare ribbons at 22:14 UT have thickened and are brighter in intensity. The current density overlays are shown in panels (c) and (e), and we selected the HMI times of 22:12 UT (b) and 22:24 UT (e) that match best.

In particular, the current density structures match the hook of the northern flare ribbon very well $\left(\right.$ at $\left.(x, y)=\left(280^{\prime \prime}, 135^{\prime \prime}\right)\right)$ : the curve of the hook, its tip structure (northern most red arrow 

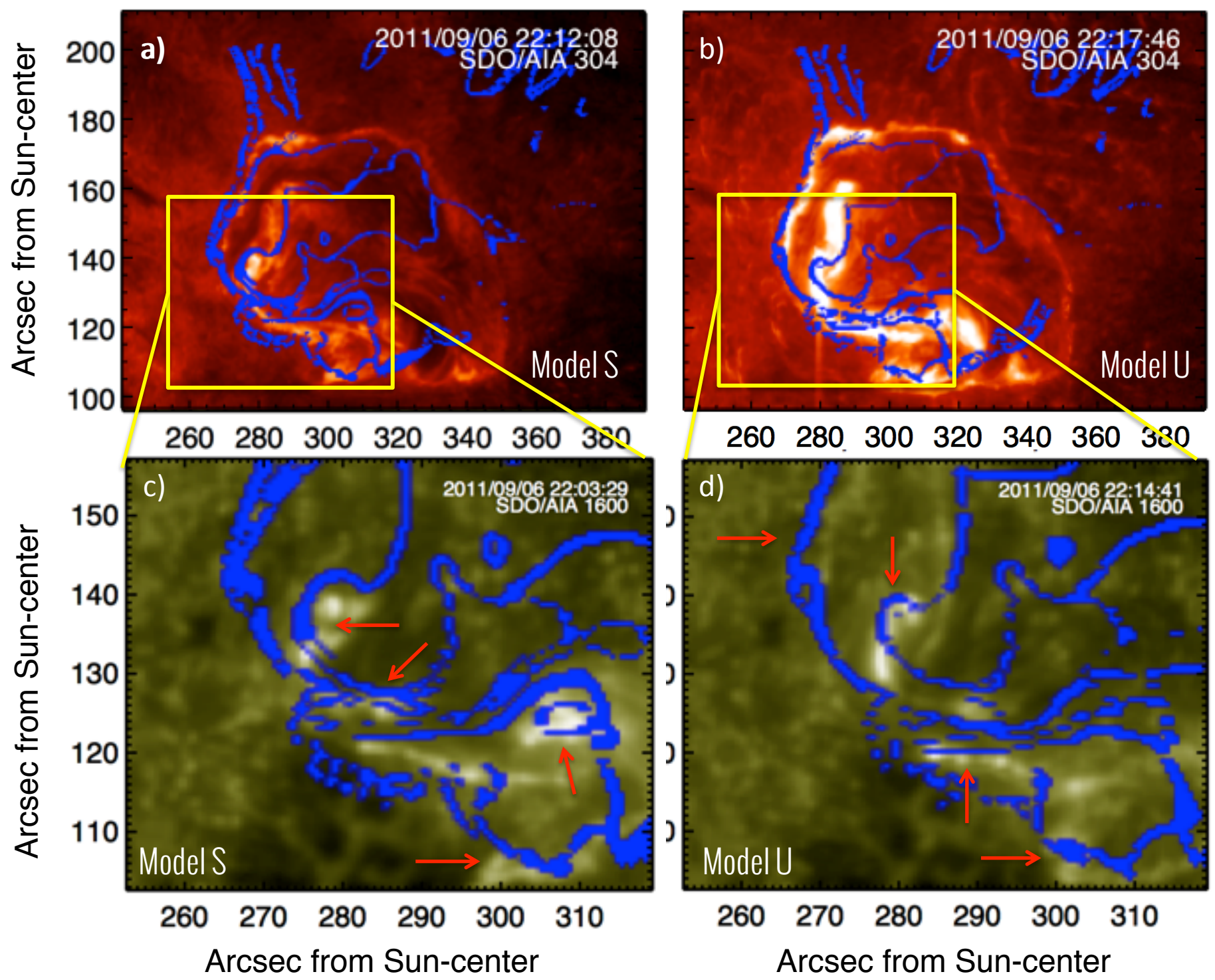

Fig. 10. Overlay of QSLs (with $Q>10^{4}$ ) obtained from the data-constrained magnetic field model (Sect. 5) with the flare ribbons seen in different filters ( $304 \AA$ and $1600 \AA$ ). a) Overview of the fan region with large circular flare ribbons best seen in $304 \AA$, matching the QSL photospheric traces for the stable model S (iteration $n=40000)$. b) Same field of view but at a later time during the peak of the flare (22:17 UT) with the QSL photospheric maps obtained for the unstable model $\mathrm{U}$ (iteration $n=30000$ ). c) Zoomed-in view of the ribbons and QSLs where the filament $\mathrm{f} 1$ is found, and where the electric current density is calculated (Fig. 3) for model S and compared with the flare ribbons seen in the $1600 \AA$, where they appear less saturated. d) The same for model U. The similarities between the ribbons and traces of QSLs in the filament region are indicated with white arrows.

in panel (b)), its thickening at 22:14 UT are all matched well, having in mind the complexity of the field. For the southern flare ribbon, found between $x=\left[280^{\prime \prime}, 310^{\prime \prime}\right], y=120^{\prime \prime}$, its elongated straight portion is also matched well by the current ribbon (as indicated with the southern most red arrow). Such a similarity between the flare and current ribbons was pointed out for the first time during an X-class flare in Janvier et al. (2014). The present analysis shows that the occurrence of current ribbons, i.e., of compact and structured high current density areas and their close relation with the flare ribbons, is a recurrent feature in filament erupting, intense magnetic field regions.

We also show in panels (c) and (f) an overlay of the flare ribbons in $1600 \AA$, the current ribbons, and with the QSLs obtained with the NLFFF method (Sect. 4) superimposed. The QSLs, with threshold $Q>10^{4}$, are the same as in Fig. 10. Similar to Fig. 11, the QSLs do not match the current ribbons exactly, although they have similarities in shape and evolution. First, the northern, positive current ribbon displays a similar shape as the QSL for the hook $\left(270^{\prime \prime}<x<280^{\prime \prime}, 130^{\prime \prime}<y<145^{\prime \prime}\right)$, while the straight part of the southern, negative current ribbon also has a QSL counterpart. They are not located at exactly the same locations: the negative ribbbon is located around $y=120^{\prime \prime}$, while the associated QSL branch is located around $y=125^{\prime \prime}$. A discrepancy in the locations of the strongest current density regions and the QSLs was also found in numerical simulations such as in Wilmot-Smith et al. (2009). Furthermore, given that the QSLs (computed at $z=4 \mathrm{Mm}$, see Sect. 5.1), the current density ribbons, which are measured in the photosphere at the depth of the Fe I absorption line at $6173 \AA$, and the flares ribbons, which appear at the height of interaction of the flare accelerated particles with the dense chromospheric plasma, are structures located at slightly different altitudes, minor horizontal shifts are expected in their shapes and locations. In addition, the difference between the time of the magnetic field measurements and the time of the 

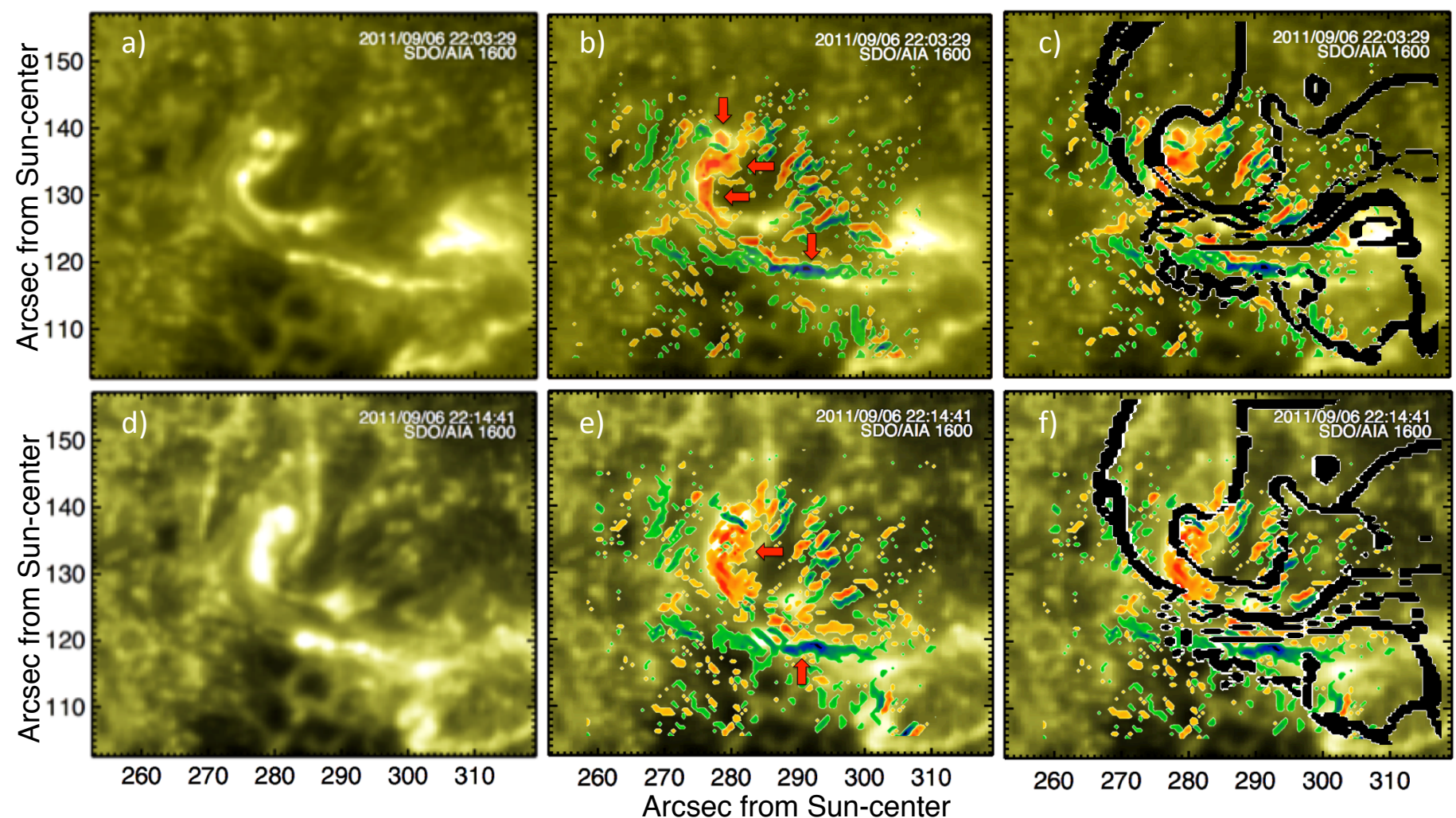

Fig. 11. Overlay of the current ribbons (see Sect. 3) on top of the flare ribbons. a) Flare ribbons obtained in the 1600 A AIA filter at 22:03 UT, well before the peak of the flare (same for d) at 22:14 UT) b) overlay with the $J_{z}$-map. Because of the HMI cadence, the $J_{z}$-map was at 22:12 UT (same for e) at 22:24 UT). The regions of strong changes, appearing both for the flare ribbons and current ribbons, are indicated with the thick red arrows. c) Overlay with QSLs (with $Q>10^{4}$ ) obtained from magnetic field models, showing the matching in the morphology of flare ribbons, QSLs, and current ribbons (same for f) at 22:24 UT).

ribbon formation can also induce a further slight discrepancy. Finally, the QSLs maps depend on an NLFFF model that does not necessarily perfectly represent the real 3D magnetic field, although bears a remarkable resemblance. However, it is striking that within a distance of a few arcsecs, the QSLs and current ribbons, which were obtained with two different techniques, are situated in similar locations and display similar morphologies. To our knowledge, this is the first time that such a detailed analysis of all these features with a high degree of matching is performed from an observation.

The QSLs show more large-scale structures than the current density. For example, the southern flare ribbon hook is nicely reproduced by the round QSL $\left(300^{\prime \prime}<x<320^{\prime \prime}, 100^{\prime \prime}<y<\right.$ $\left.130^{\prime \prime}\right)$ while the current density is well below the noise level at this location. As shown in Fig. 10, the QSLs also extend northward and reproduce well the large-scale circular ribbon, while the current ribbons are only located where the filament f1 is situated.

\section{Unstable model evolution}

\subsection{Flux rope associated QSL evolution}

The two flare ribbons associated with a flux rope ejection typically move away from each other as the flare evolves. We find a similar behavior for the ribbons associated with the ejection of filament f1, as shown in Fig. 12. In the three top panels, we show three AIA observations at different times: well before (left), during (middle), and after (right) the flare peak. The separation of the flare ribbons is indicated with a double red arrow, while different portions of the evolving ribbons are shown with orange arrows. The straight part of the northern ribbon, found at $y \approx 125^{\prime \prime}$, has moved up to $y \approx 130^{\prime \prime}$ at 22:27 UT. We also find that the hook portion of the northern ribbon (at $y=130^{\prime \prime}$ ) moves slightly westward, starting at $x=275^{\prime \prime}$ at 22:03 UT up to $x=282^{\prime \prime}$. The hook of the southern ribbon becomes rounder at 22:14 UT, while the straight portion, found at $y \approx 117^{\prime \prime}$ at 22:03 UT, has moved southward at position $y \approx 105^{\prime \prime}$.

Then, these different observations can be compared with the different iterations of the unstable model $\mathrm{U}$, obtained with the magnetofrictional code. Although the MF process cannot correctly reproduce the dynamics of the filament eruption in the same way that an MHD simulation would, it reproduces the evolution of the topology very well. Then, the MF process provides valuable insights into the evolution of the QSLs and the magnetic field, which can then be compared with the observations. For example, we find the following similarities with the observations: an outward motion of the QSLs away from the inversion line (also indicated with the double red arrow). At iteration 55000 , the central QSLs are largely separated from each other. At the same time, the hook region of the QSLs associated with the flux rope is evolving in a similar fashion as the flare ribbons; the northern ribbon hook moves westward. The three iterations indicate a good agreement between the different positions and shapes of the flare ribbons and corresponding QSLs. The northern ribbon hook is also seen to shrink as time goes by, indicating that the flux rope may end up anchored in only one polarity. This is justified by the behavior of some magnetic field lines associated with the flux rope that leave the dome and hint at a long-range interaction (see Sect. 6.2). Finally, the hook of the southern branch of the QSL also becomes rounder at iteration 

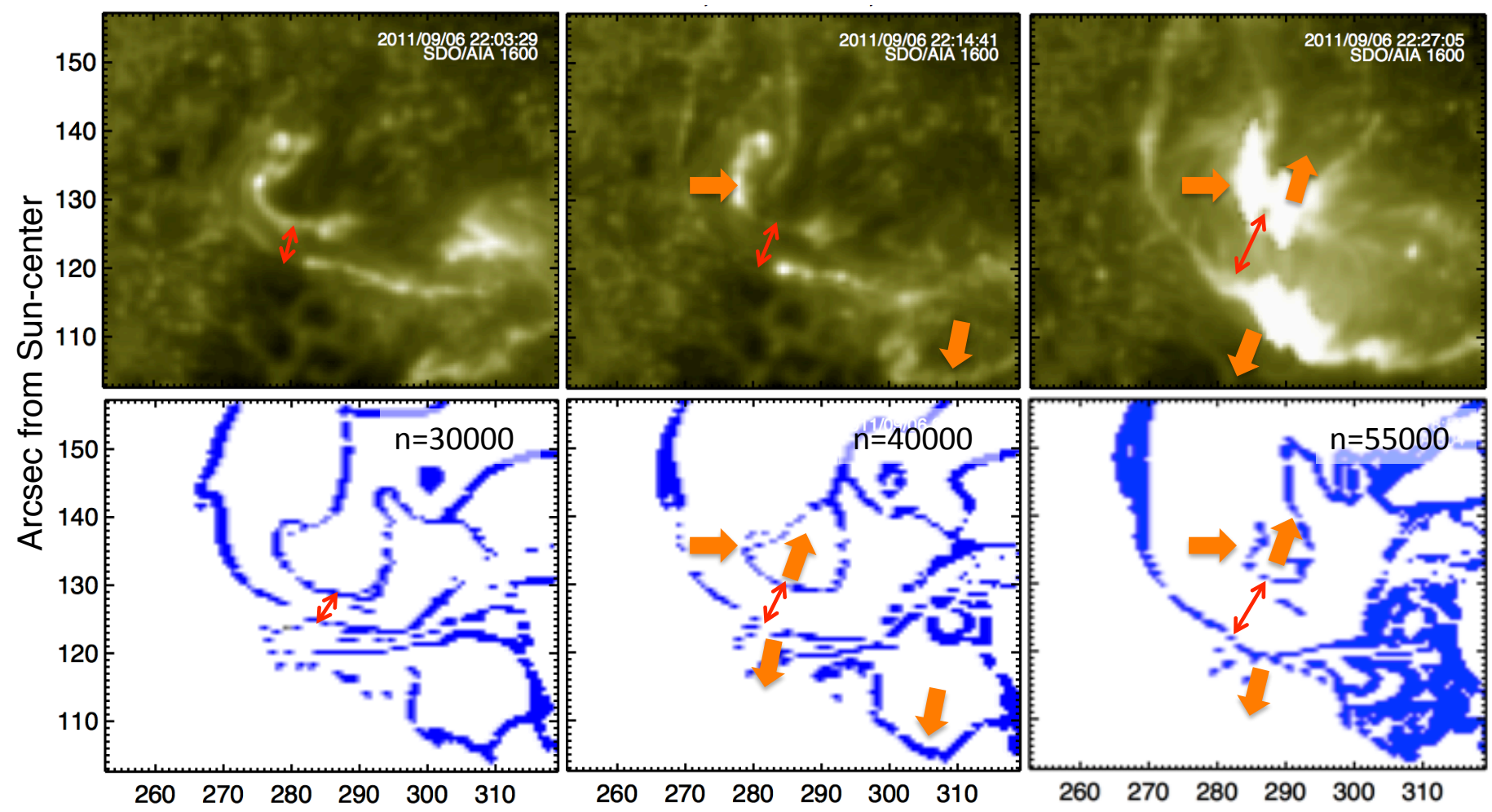

Arcsec from Sun-center

Fig. 12. Top: flare ribbons seen in the $1600 \AA$ filter of AIA. The field of view is zoomed on the filament f1 (Fig. 1a) and is the same as the area where the electric current density is calculated in Fig. 3. Three different times are shown for the evolution of the ribbons. The strong emissions show highly structured ribbons. Bottom: QSL maps at $z=4 \mathrm{Mm}$ at different iterations from the magnetofrictional evolution of model U. Similar to flare ribbons, the central parts of the QSLs are seen to move away from each other (indicated by the double red arrow). Different portions of the QSLs are also evolving, as indicated with the thick orange arrows, similar to the flare ribbons.

40000 (arrow shown at $x=305^{\prime \prime}, y=105^{\prime \prime}$ ). One main difference, however, is that this southern QSL does not move to the same latitude as its flare ribbon counterpart. Such a spreading of the low-lying footprints (those under the HFT) of the flux rope binding QSLs, which matches the perpendicular motion of flare ribbons in three two-ribbon flares, was first shown in Savcheva et al. (2016) in Figs. 13-15.

\subsection{Filament eruption}

The evolving model $\mathrm{U}$ allows us to trace the changes in the connectivity of the magnetic field lines. Several iterations of this evolution are shown in Fig. 13, where some sample field lines are chosen to depart from the filament $\mathrm{f} 1$ region. At $n=40000$, we present a zoom of the region where the most intense emission of the flare occurs. There we see field lines that are anchored in the southwestern $J$ and leave the simulation box. The direction in which they leave is not the direction of the spine, but rather the direction of the filament eruption as can be seen from panel (f) of Fig. 1. One can see from later iterations and with a larger zoom $(n=55000,120000$ and 150000$)$ that field lines traced from the same locations are seen to connect far from the active region to the north. In particular, a group of field lines connect to the northwest region of weak positive magnetic field. This direction of the filament eruption and the subsequent partial raining down of the filament material in the exact same direction is studied by means of a data-constrained MHD simulation by Jiang et al. (2014b).
Although Fig. 13 does not present a proper MHD simulation, the code used employs magnetofriction and the opening of field lines associated with the flux rope can show the path taken by the flux rope during its eruption. For example, Lugaz et al. (2011) showed that a flux rope embedded in a complex topological domain such as a null point dome could result upon eruption in a mixing of closed and open field lines, where the open field lines can give way to the filament eruption (see, e.g., Fig. 5 in their paper). Here, the large-scale magnetic field also provides such a clue.

\subsection{Long-range interaction of the magnetic field}

Figure 13 shows another group of field lines connecting to the eastern side of the active region, where the faculae region is found (see Fig. 2). In the observations, especially in the $335 \AA$ filter of AIA, a strong remote bright ribbon is found in the faculae region, surrounding a dimming (panel e of Fig. 1), while the AIA $171 \AA$ filter at 22:40 UT (panel f of Fig. 1) shows long loop arcades connecting the faculae region to the core of the active region, where the two filament eruptions occur.

Similarly, model U hints at a long-range interaction. We see field lines connecting over the entire positive faculae region to the east of the sigmoid. The upper right panel, in fact, shows several arcade field lines that connect the edge of the flux rope with the western half of the remote ribbon. However, there are plenty of long loops found at other locations that are not anchored exactly in the same region as the remote brightening seen 

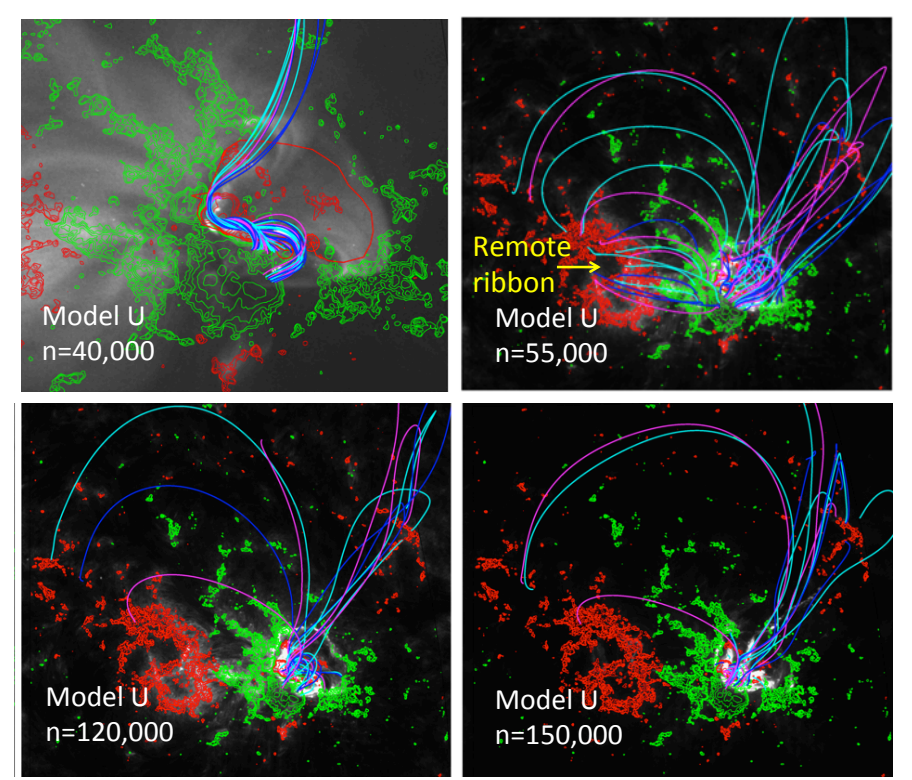

Fig. 13. Selected field lines around the flux rope for the unstable model U. The four panels represent different iterations of the magnetofrictional relaxation. The green and red contours show the magnetogram of Fig. 2 overplotted on four AIA images (gray scale) showing the bright ribbons in the $304 \AA$ filter. The iteration at $n=40000$ was zoomed in so as to show parts of the field lines escaping from the dome region. The other iterations are shown as a full field of view of the region, showing the different loops connecting to the bipole where the eruption takes place. The selected field lines both show the extension to a north and west location with a similar orientation as the ejection of filaments $\mathrm{f} 1$ and $\mathrm{f} 2$, seen in Fig. 1. In that location, there is an extension on the eastern side, hinting at a long-range interaction with the faculae region, and some loops connecting the remote ribbon brightening.

in the observations. This long-range interaction can also be seen in Fig. 14, panels (a) and (b), where we plotted the 3D QSL maps for model $\mathrm{U}$ (iteration $n=40000$ ). These were computed on graphics processor units that allow for the massively sped-up calculations of the QSLs resulting in a million field lines with known $Q$ in a minute. The method for computing the 3D QSLs is described in detail in Tassev \& Savcheva (in prep.) and is shown for the first time here for the study of an eruptive flare. In the figure, the bottom boundary of the 3D computation is shown at a height of $4 \mathrm{Mm}$ and the 3D volume filling QSLs are displayed semitranslucently with the same color scale from one view to another.

In Figs. 14c and d, we also show 2D QSLs at the same height as before with some selected field lines (Fig. 8). In particular, the zoomed-out views of panels (b) and (d) show how the spine (red field line) emanating from the NP found in model U, connects to the easternmost part of the computation box corresponding to the regions where we also find the highly arched QSLs connecting to the lower $x$-values domain. The fact that we found a QSL connecting further away from the fan dome, in a location reminiscent of that of the remote brightening surrounding the dimming region (see panel (c) in Fig. 1), points to a typical null point flaring configuration (Masson et al. 2009). We also see that there is a stronger QSL that is sharp and narrow and connects the edge of the flux rope domain to the remote ribbon location. However, the footprint of these QSLs is not circular as seen in the observations but is rather elongated, as can be seen from the 2D QSL cut shown on the bottom boundary of the 3D box. This is not very surprising since the shape and location of remote brightenings and the direction of the spine in circular ribbon flares require very careful NLFFF modeling that is sensitive to very delicate features in the magnetic field that can have an effect on the end result.

\section{Summary}

Solar eruptive flares release coronal mass ejections and high energy particles. The developments of theoretical and numerical models of these phenomena can now be tested with ever increasing spatial and temporal resolutions of solar instruments. In the present paper, we investigate the underlying mechanism of a complex-topology, X-class flare accompanied by filament ejections using different instruments aboard SDO and XRT; we compare the evolution of this flare with that predicted by the recently developed standard model for eruptive flares in 3D (Aulanier et al. 2012; Janvier et al. 2013, 2014; Savcheva et al. 2016).

To do so, we investigated the evolution of the 6 September 2011 X-class flare occurring in region AR 11283. The AIA filters show different features, such as filaments, sigmoid, and ribbons, during the flare evolution. These are reported in Sect. 2. Using HMI data and applying an inversion method to obtain the vector magnetograms, we deduce the temporal maps of the vertical current density component and the evolution of the integrated current intensity (Sect. 3). We also use the magnetograms and the AIA images to obtain the 3D magnetic field structure of the active region from the flux rope insertion method (Sect. 4), which provides a stable model (model S) and an unstable model (model $U$ ) by adding a unit of axial flux. The modeled magnetic field is used to perform a calculation of the quasi-separatrix layers (Sect. 5.1), which is then compared with the location of the flare ribbons (Sect. 5.2) and current ribbons (Sect. 5.3). Finally, we also investigate how the different iterations of the unstable model can be compared with the evolution of the flare ribbons and other observational features in Sect. 6. The results are summarized below.

This region provides a very different magnetic field configuration study from that of the 15 February 2011 X-class flare studied in Janvier et al. (2014) and, as such, allows us to test the predictions of the 3D standard flare model in a more complex configuration. This can be gleaned from the AIA images with the presence of two parts of an elongated filament, which we refer to as filaments $\mathrm{f} 1$ and $\mathrm{f} 2$. The flare emission is accompanied by the ejection of those two parts at different times, and the presence of several ribbons (circular ribbons, two ribbons near the f1 location, and a remote ribbon in the following polarity) points toward the presence of a fan and spine configuration (Fig. 1). This is confirmed by a look at the magnetogram (Fig. 2), which shows the presence of a parasitic polarity in a decaying active region, which is also revealed by a thorough investigation of the magnetic field configuration from an extrapolation (Sect. 4).

The evolution of the current ribbons obtained with the high temporal cadence of the HMI instrument before and after the flare show different regions of current density increase (Fig. 3). These regions correspond to a broadening, elongation, and a separation away from the PIL of the current ribbons. The integration of the current density shows that the electric current increases during the impulsive phase of the flare, and remain at high levels after the flare peak (Fig. 4). These results confirm the first observational analysis for an X-class flare published in Janvier et al. (2014) and the predictions of the current layer evolution during an eruptive flare from the 3D flare model (Aulanier et al. 2012; Janvier et al. 2013). 

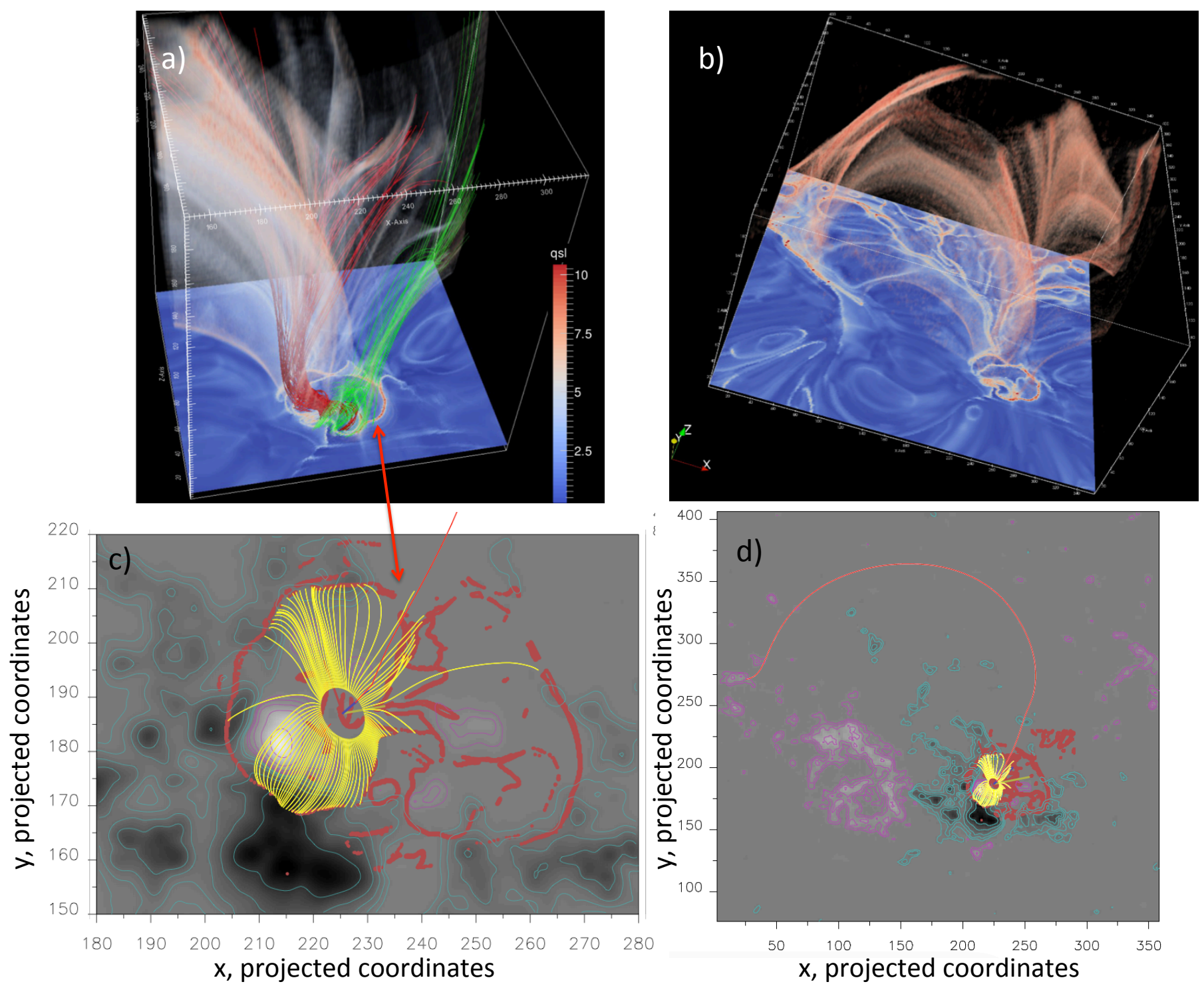

Fig. 14. a) Three-dimensional QSL computation obtained for iteration $n=40000$ of model $\mathrm{U}$ zoomed in on the circular ribbon region; the redder the QSLs the stronger they are. The maximum value of $Q$ shown is $10^{10}$. It shows the different QSL volumes departing from the dome region and the null point. In particular, a set of QSLs extend in the direction of the spine. The red field lines are flux rope field lines that leave the dome after they go through a bald patch area, i.e., a region where dipped field lines are tangent to the photospheric boundary; Titov et al. (1993), as described also in Fig. 13. b) The same QSLs are plotted in a more global view showing that some 3D QSLs, which depart from the fan region on the western side, connect to the remote brightening on the eastern side. Specifically, a set of QSLs connect the flux rope to the western edge of the fan region to the remote brightening. On the bottom surface of both 3D plots we have shown the 2D horizontal map at $z=4 \mathrm{Mm}$ with the same color scaling. c) 2D map of the QSLs at $z=4 \mathrm{Mm}$ from the Pariat \& Démoulin (2012) calculation technique, zoomed in on the circular ribbon region. The yellow lines are the fan of the null point, while the red lines show the spine. d) This zoomed out 2D map shows the QSL signature; the spine red field line connects to the eastern area of the active region.

We performed an NLFFF modeling with the flux rope insertion method. The best-fit stable model $\mathrm{S}$ indicates that a flux rope only exists over the southern part of the PIL, where most of the twisted field lines are concentrated and the sigmoid in seen the AIA $131 \AA$ A channel and XRT. The unstable model U, obtained by increasing the axial flux of the best-fit NLFFF model S by one unit, is evolved via magnetofriction (Fig. 6). Although this process is not a real MHD evolution, it remarkably succeeds at rendering the evolution of the magnetic field and its topology as the flux rope erupts (see Fig. 7c). In particular, we confirm that there is a null point that is confirmed by the presence of fan and spine field lines. The QSLs calculations for both models S and $\mathrm{U}$ display a circular QSL, as would be expected in the presence of a null point-associated dome (Fig. 8). Tracing the field lines associated with the flux rope allows us to pinpoint their associated QSLs; they display a $J$ shape, as predicted by the 3D flare model (Fig. 9).

The QSLs evolution in the different iterations of model $\mathrm{U}$ and the evolution of the current ribbons are directly compared with the EUV flare ribbons. The QSLs computed at different iterations display the same features as found in the observations: these are particularly good for the large-scale ribbons see in the $304 \AA$ AIA filter (Fig. 10, top panels). They are also well localized for the ribbons associated with the erupting filament $\mathrm{f} 1$, best seen in the $1600 \AA$ A filter (Fig. 10, bottom panels). These different iterations also reproduce the motion of the ribbons away 
from the inversion line and the evolution of their morphlogy, as shown in Fig. 12.

Overlaying the flare ribbons (from AIA images) and the current ribbons (derived from HMI data) also show a very good correspondence in the location of the intense emissions for the ribbons and high current density patches for the current ribbons (Fig. 11, middle column). This is a particularly interesting result since the complex topology could have strongly affected the predicted shape of the current ribbons. Although in the present case, we do not find two $J$-shaped current ribbons (this shape is only found for the positive northern current ribbon), their correspondence to the flare ribbons and the QSLs, and their evolution in time, show that these are stable features that should be found in most flares, provided that the magnetic field is strong enough to derive a current density value that is higher than the noise ratio.

We looked at the evolution of the QSLs and the magnetic field lines computed from the modeled magnetic field at different iterations in the relaxation runs of model U. In particular, the evolution of the large-scale magnetic field hints at a longrange interaction. On the one hand, magnetic field lines connect from the filament eruption site to a site further to the north and west, indicating a possible escape route for the filaments seen to erupt in the same direction (Fig. 1, panels $d$ and f). On the other hand, other magnetic field lines connect to the eastern faculae region, where a strong remote brightening and dimming were found. Plotting the 3D volume of the QSLs (Fig. 14) with a novel technique (Tassev \& Savcheva, in prep.), we saw that the edge of the spine region of the null point connects to this region, although the locations of the spine footpoint and brightening seen in the observations remain far away from each other.

\section{Conclusions}

Flare ribbons are typically interpreted as strong heating taking place at the chromospheric levels due to interaction between the ambient plasma and the energetic particles that have been accelerated from the reconnection site, higher up in the corona (Reid et al. 2012). This is the outcome of a magnetic field dissipation process, called reconnection, taking place in a high current density layer. This 3D coronal current layer cannot be resolved by today's instrumentation, however, as it extends toward the lower levels of the Sun's atmosphere (see Fig. 7 in Janvier et al. 2014), its evolution can be recorded by the changes in the horizontal components of the photospheric magnetic field.

In the presence of a flux rope, the shape and locations of current and EUV ribbons follow that of the footprints of the fluxrope-binding QSL and are all expected to have a double $J$-shaped structure. This was shown to be the case in an X-class flare occurring in a simple bipole (Janvier et al. 2014). The present flare provides other evidence of the current density strongly evolving during a flaring event: an increase that persists after the end of the flare and coincides with the impulsive phase. Furthermore, the active region studied in this paper presents a much more complex organization of the magnetic field; the erupting filament is embedded in a magnetic dome due to the presence of a null point. As such, we have seen that one of several flare ribbons extends far north with a circular morphology (Fig. 10). Nonetheless, the present results show that even in the presence of a complex magnetic field configuration, the current ribbons display a $J$-shaped structure, although this structure can be altered by the surrounding configuration (the hook of the southern current ribbon is missing).

Then, the present paper provides for the first time a joint analysis of the evolution of the photospheric current ribbons and the QSLs deduced from observations with SDO instruments. Although the current ribbons are deduced from HMI data and the QSLs are computed from a magnetic field model, we gave (Fig. 11) direct evidence of the changes in the electric current layer during the impulsive phase of the flare; these changes are indeed associated with similar changes in the flare ribbons and QSLs.

Hence, the good agreements in the different methods suggest that the predictions given by the standard model in 3D for eruptive flares (see Fig. 11 in Janvier et al. 2015) are stable features that ought to be seen in a variety of magnetic field configurations associated with a flux rope eruption.

More insight in the topology of flares and its connection with the current layers will be given with future instruments with better spatial resolution and looking at different coronal heights. This is in particular of great interest to connect the behavior of the photospheric and chromospheric signatures with that of the corona, both for the current layer and magnetic field (QSLs). Such knowledge will be fundamental to better understand the physical mechanisms of the current layers, where the magnetic field is ultimately dissipated during flares.

Acknowledgements. We thank the SDO/HMI and SDO/AIA teams for providing the data. A.S. thanks E. DeLuca for fruitful discussions. This work was partially supported by the Northern Research Partnership (at University of Dundee, UK) grant and by a one-month invitation of M.J. to the Harvard-Smithsonian Astrophysical Observatory and a short visit of A.S. at the University of Dundee, UK This work (magnetogram inversions) was granted access to the HPC resources of MesoPSL financed by the Region Île de France and the project Equip@ Meso (reference ANR-10-EQPX-29-01) of the programme Investissements d'Avenir supervised by the Agence Nationale pour la Recherche.

\section{References}

Aulanier, G., Pariat, E., Démoulin, P., \& DeVore, C. R. 2006, Sol. Phys., 238, 347

Aulanier, G., Janvier, M., \& Schmieder, B. 2012, A\&A, 543, A110

Bommier, V., Landi Degl'Innocenti, E., Landolfi, M., \& Molodij, G. 2007, A\&A, 464, 323

Chandra, R., Schmieder, B., Aulanier, G., \& Malherbe, J. M. 2009, Sol. Phys., 258, 53

Chen, P. F. 2011, Liv. Rev. Sol. Phys., 8

Démoulin, P. 2006, Adv. Space Res., 37, 1269

Démoulin, P., Hénoux, J. C., Priest, E. R., \& Mandrini, C. H. 1996a, A\&A, 308, 643

Démoulin, P., Priest, E. R., \& Lonie, D. P. 1996b, J. Geophys. Res., 101, 7631

Emslie, A. G., Kontar, E. P., Krucker, S., \& Lin, R. P. 2003, ApJ, 595, L107

Feng, L., Wiegelmann, T., Su, Y., et al. 2013, ApJ, 765, 37

Fletcher, L., Dennis, B. R., Hudson, H. S., et al. 2011, Space Sci. Rev., 159, 19

Gary, G. A., \& Démoulin, P. 1995, ApJ, 445, 982

Gary, G. A., Hu, Q., Lee, J. K., \& Aschwanden, M. J. 2014, Sol. Phys., 289, 3703

Golub, L., Deluca, E., Austin, G., et al. 2007, Sol. Phys., 243, 63

Gonzalez, W. D., Tsurutani, B. T., \& Clúa de Gonzalez, A. L. 1999, Space Sci. Rev., 88, 529

Gosain, S., Démoulin, P., \& López Fuentes, M. 2014, ApJ, 793, 15

Gosling, J. T., McComas, D. J., Phillips, J. L., \& Bame, S. J. 1991,

J. Geophys. Res., 96, 7831

Green, L. M., \& Kliem, B. 2009, ApJ, 700, L83

Green, L. M., Kliem, B., \& Wallace, A. J. 2011, A\&A, 526, A2

Hudson, H. S., Wolfson, C. J., \& Metcalf, T. R. 2006, Sol. Phys., 234, 79

Janvier, M., Aulanier, G., Pariat, E., \& Démoulin, P. 2013, A\&A, 555, A77

Janvier, M., Aulanier, G., Bommier, V., et al. 2014, ApJ, 788, 60

Janvier, M., Aulanier, G., \& Démoulin, P. 2015, Sol. Phys., 290, 3425

Jiang, C., \& Feng, X. 2013, ApJ, 769, 144

Jiang, C., Feng, X., Wu, S. T., \& Hu, Q. 2013, ApJ, 771, L30

Jiang, C., Wu, S. T., Feng, X., \& Hu, Q. 2014a, ApJ, 780, 55

Jiang, C., Wu, S. T., Feng, X., \& Hu, Q. 2014b, ApJ, 786, L16

Kliem, B., Su, Y. N., van Ballegooijen, A. A., \& DeLuca, E. E. 2013, ApJ, 779, 129

Koleva, K., Madjarska, M. S., Duchlev, P., et al. 2012, A\&A, 540, A127 
Leka, K. D., Barnes, G., Crouch, A. D., et al. 2009, Sol. Phys., 260, 83 Lemen, J. R., Title, A. M., Akin, D. J., et al. 2012, Sol. Phys., 275, 17

Liu, C., Deng, N., Lee, J., et al. 2014, ApJ, 795, 128

Liu, C., Deng, N., Liu, R., et al. 2015, ApJ, 812, L19

Longcope, D. W. 2005, Liv. Rev. Sol. Phys., 2, 7

Lugaz, N., Downs, C., Shibata, K., et al. 2011, ApJ, 738, 127

Malanushenko, A., \& Savcheva, A. 2016, Frontiers in Space Science, submitted

Masson, S., Pariat, E., Aulanier, G., \& Schrijver, C. J. 2009, ApJ, 700, 559

Musset, S., Vilmer, N., \& Bommier, V. 2015, A\&A, 580, A106

Pariat, E., \& Démoulin, P. 2012, A\&A, 139, A78

Petrie, G. J. D. 2012, ApJ, 759, 50

Prangé, R., Pallier, L., Hansen, K. C., et al. 2004, Nature, 432, 78

Priest, E. R., \& Démoulin, P. 1995, J. Geophys. Res., 100, 23443

Priest, E. R., \& Forbes, T. G. 2002, A\&ARv, 10, 313

Reid, H. A. S., Vilmer, N., Aulanier, G., \& Pariat, E. 2012, A\&A, 547, A52

Rust, D. M., \& Kumar, A. 1996, ApJ, 464, L199

Savcheva, A., \& van Ballegooijen, A. 2009, ApJ, 703, 1766

Savcheva, A., Pariat, E., van Ballegooijen, A., Aulanier, G., \& DeLuca, E. 2012a, ApJ, 750, 15

Savcheva, A. S., Green, L. M., van Ballegooijen, A. A., \& DeLuca, E. E. 2012b ApJ, 759, 105

Savcheva, A. S., van Ballegooijen, A. A., \& DeLuca, E. E. 2012c, ApJ, 744, 78

Savcheva, A., Pariat, E., McKillop, S., et al. 2015, ApJ, 810, 96

Savcheva, A., Pariat, E., McKillop, S., et al. 2016, ApJ, 817, 43

Scherrer, P. H., Schou, J., Bush, R. I., et al. 2012, Sol. Phys., 275, 207

Schou, J., Scherrer, P. H., Bush, R. I., et al. 2012, Sol. Phys., 275, 229

Solanki, S. K., \& Montavon, C. A. P. 1993, A\&A, 275, 283

Su, Y., Surges, V., van Ballegooijen, A., DeLuca, E., \& Golub, L. 2011, ApJ, 734, 53

Thomas, J. H., \& Weiss, N. O. 2012, Sunspots and Starspots (Cambridge, UK: Cambridge University Press, 2012)

Titov, V. S. 2007, ApJ, 660, 863

Titov, V. S., \& Démoulin, P. 1999, A\&A, 351, 707

Titov, V. S., Priest, E. R., \& Demoulin, P. 1993, A\&A, 276, 564

Titov, V. S., Hornig, G., \& Démoulin, P. 2002, J. Geophys. Res., 107, 1164

van Ballegooijen, A. A. 2004, ApJ, 612, 519

Wang, S., Liu, C., \& Wang, H. 2012, ApJ, 757, L5

Wang, H., Liu, C., Deng, N., et al. 2014, ApJ, 781, L23

Wheatland, M. S., \& Gilchrist, S. A. 2013, J. Phys. Conf. Ser., 440, 012037
Wilmot-Smith, A. L., Hornig, G., \& Pontin, D. I. 2009, ApJ, 704, 1288

Xu, Y., Jing, J., Wang, S., \& Wang, H. 2014, ApJ, 787, 7

Yang, Y.-H., Chen, P. F., Hsieh, M.-S., et al. 2014, ApJ, 786, 72

Yang, K., Guo, Y., \& Ding, M. D. 2015, ApJ, 806, 171

Zhang, Q. M., Ning, Z. J., Guo, Y., et al. 2015, ApJ, 805, 4

Zhao, J., Li, H., Pariat, E., et al. 2014, ApJ, 787, 88

Zhao, J., Gilchrist, S. A., Aulanier, G., et al. 2016, ApJ, 823, 62

Zharkov, S., Green, L. M., Matthews, S. A., \& Zharkova, V. V. 2013, J. Phys. Conf. Ser., 440, 012046

\section{Appendix A: Best-fit NLFFF stable model}

We experimented with three different paths over which the flux rope is inserted because the AIA $304 \AA$ observations seemed to show the presence of two filaments that could be part of the same structure (Fig. 1a). Indeed, they both extend along the whole PIL around the parasitic polarity and not only where the sigmoid is observed (as shown in Fig. 1b). This method therefore allows us to look at paths that include only f1 or both $\mathrm{f} 1$ and $\mathrm{f} 2$ to choose the most stable result and, as such, is different from those proposed by different authors (Feng et al. 2013; Jiang et al. 2013; Liu et al. 2014) in previous studies.

These paths are shown in Fig. A.1. In the left panel, we showed the path that corresponds only to the filament underlying the sigmoid, shown on top of the current distribution obtained in the best-fit model for the given path. The middle panel shows an almost circular path with a "barb" inserted on the left side of the sigmoid (indicated in blue colors). The purpose of the barb is to reduce the flux in the flux rope beyond this point, so essentially we have a flux rope with variable axial flux along its length. The right panel shows the path that produced the best-fit model (hereafter referred to as model S). It follows a circular path with constant axial flux.
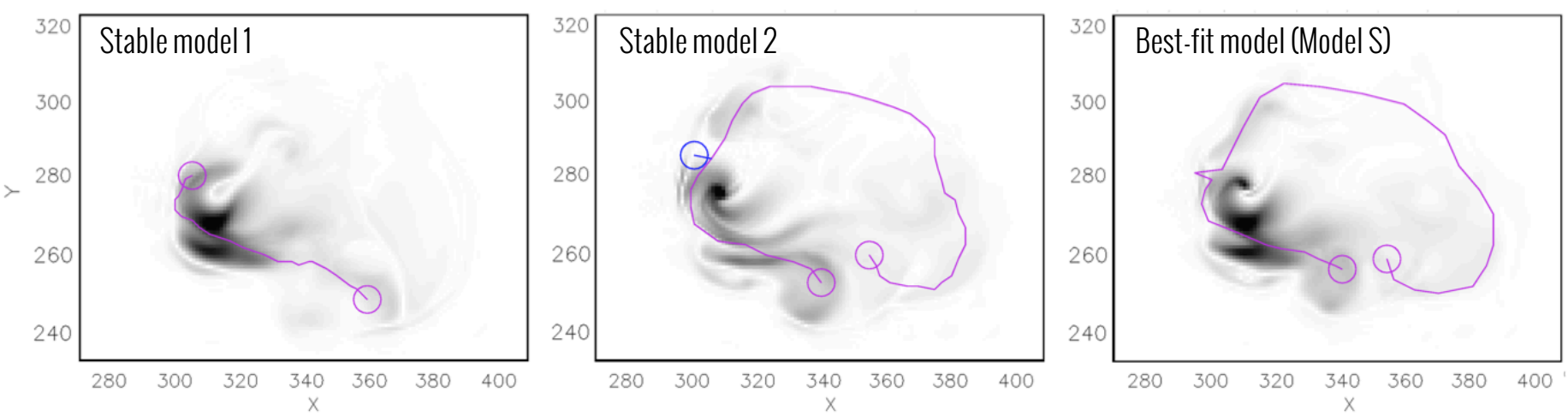

Fig. A.1. Different chosen paths (in purple) for the flux rope insertion method. The black and white color scale represents the modeled current density distribution. Left: path was aligned with the filament underlying the sigmoid, as seen in the $131 \AA$ A filter (Fig. 1a). Middle: circular path extending the filament, corresponding to a flux rope with a variable axial flux, as represented by the blue insert on the left of the sigmoid. Right: best-fit model corresponding to a flux rope with a constant axial flux, referred to as model S. 